\title{
On Dielectronic Recombination
}

\author{
S. M. R. ANSARI *, G. Elwert, and P. MÜCKLICH * * \\ Lehrstuhl für Theoretische Astrophysik der Universität Tübingen, Germany
}

(Z. Naturforsch. 25 a, 1781-1797 [1970] ; received 20 June 1970)

\begin{abstract}
The derivation of a general formula for the coefficient of dielectric recombination is reviewed, and approximate formulae known in the literature are discussed. With the aid of Burgess' simplified formula calculations of the coefficient of dielectronic recombination for the elements $\mathrm{C}, \mathrm{O}, \mathrm{Ne}, \mathrm{Mg}$, $\mathrm{Si}, \mathrm{S}$ and $\mathrm{Fe}$ in the temperature range $10^{6}-4 \times 10^{6}{ }^{\circ} \mathrm{K}$ are performed. The results are presented in figures and in tabular form. Taking into account dielectronic and radiative recombination, the ionization equilibrium for the various stages of these elements is determined and compared with recent results of other authors.
\end{abstract}

\section{Introduction}

The contribution of dielectronic recombination to the ionization equilibrium of the solar corona reduces the appreciable discrepancy between its temperature as deduced from the observational Doppler line-broadening and that calculated on the basis of the ionization theory. This fact has been conjectured by Unsöld and was fairly corroborated by calculations of BURGESS ${ }^{1-3}$ and SEATON ${ }^{3}$. In addition to the application of this process to solar physics, it has grown in importance generally and its astrophysical applications are multiplying (WIDING ${ }^{4}$, TuCker and Gould ${ }^{5}$, Elwert ${ }^{6}$, POtTash ${ }^{7}$, Evans and Pounds ${ }^{8}$ ). It for instance influences the radio recombination lines (GAYET et al. ${ }^{9}$ ) and plays a role in the planetary nebulae and in H II regions (Goldberg ${ }^{10}$, Goldberg and Dupree ${ }^{11}$ ).

In the following, the general formula of the coefficient of dielectronic recombination $\alpha_{\mathrm{di}}$ is derived

Reprints request to Prof. Dr. G. Elwert, Lehrstuhl für Theoretische Astrophysik der Universität Tübingen, D-7400 Tïbingen, Hausserstraße 64.

* Present address: Department of Physics, Aligarh Muslim University, Aligarh (U.P.), India.

* Present address: IBM, Sindelfingen, Germany.

1 A. Burgess, Astrophys. J. 139, 776 [1964].

2 A. Burgess, General Formula of Estimating Dielectronic Recombination Coefficient (distributed privately) 1964.

3 A. Burgess and M. J. Seaton, Mon. Not. Roy. Astr. Soc. 127, 355 [1964].

4 K. G. WIDING, Astrophys. J. 143, 606 [1966].

5 W. H. TuCKer and R. J. Gould, Astrophys. J. 144, 244 [1966].

6 G. ElWert, Space Research VII, p. 1287, North Holland Publ. Co. 1967.

7 S. R. Pott ash, Bull. Astr. Inst. Netherlands 19, 113 [1967].

8 K. Evans and K. A. Pounds, preprint, Univ. of Leicester 1967.

9 R. Gayet, D. Hoang Binh, F. Joly, and R. McCarrol, Astron. Astrophys. 1, 365 [1969]. and a few approximate expressions for $\alpha_{\mathrm{di}}$ are briefly reviewed. We endeavor to point out the physical assumptions as clearly as possible, because we think that, due to a practical standpoint, this has been neglected so far in the literature.

Calculations of $\alpha_{\mathrm{di}}$ especially for ions abundant in the solar corona using Burgess' simplified formula (BURGEss ${ }^{12,13}$ ) are presented ${ }^{14}$; the limitations of the Burgess formula are particularly stressed. For low density plasmas this expression is the best possible one, up to now. It allows to calculate $\alpha_{\mathrm{di}}$ without great difficulty and to an accuracy presently sufficient for most of the ions important in astrophysical applications, especially in the solar corona and in similar low density plasmas (BURGESS and SUMmers ${ }^{18}$ ). Consequently, values of $\alpha_{\mathrm{di}}$ calculated by the Burgess formula are published here without any modification (Section 6). It is intended to sketch in a forthcoming publication the more modern quantum mechanical treatment of the

10 L. Goldberg, Scientific Report No. 25, Harvard College Observatory, February 1968, or see Interstellar Ionized Hydrogen: Proceedings of NRAO-AIO H II Region Symposium, ed. Y. Terzian, W. A. Benjamin Inc., New York 1968.

11 L. Goldberg and A. K. Dupree, Nature London 215, 41 [1967].

12 A. Burgess, Astrophys. J. 141, 1588 [1965].

13 A. Burgess, IAU-Symposium No. 23, Astronomical Observations from Space Vehicles, Ed. J.-L. STEINBERG, p. 95/96, or see Ann. d'Astrophysique 1965, No. 1-6.

14 The numerical results given here have partly been reported on earlier meetings (ANSARI et al. ${ }^{15}$, MǗCKLICH ${ }^{16}$, ELWERT ${ }^{17}$ ).

15 S. M. R. Ansari. G. Elwert, and P. Mücklich, Mittlgn. Astron. Ges. No. 25, p. 200 [1968].

16 P. MÜCKLICH, Diplomarbeit, University of Tübingen 1966.

17 G. Elwert, in: Physics of the One- and Two-Electron Atoms, Ed. F. Bopp and H. KLeINPOPPEN, North Holland Publ. Comp., Amsterdam 1969.

18 A. Burgess and P.Summers, Astrophys. J. 157, 1007 [1968]. 
process (ShORE ${ }^{19}$, TRefFTZ ${ }^{20}$, DAvies and SeATON ${ }^{21}$ ). The minor modification caused in the values of $\alpha_{\mathrm{di}}$ by autoionization from collisionally excited states and by depopulation of the highly excited levels of the captured electron by collisions in case of dense plasmas is intended to be treated in the next paper.

Instead of tabulating $\alpha_{\mathrm{di}}$ for various ions and for various temperatures $T$, a reduced formula for $\alpha_{\mathrm{di}}(T)$ is given as a sum of a few terms whose parameters are tabulated for important ions. Calculations of the ionization equilibrium taking into account dielectric recombination are also performed and compared with the results of JORDAN ${ }^{22}$ and with those obtained by using the formula for $\alpha_{\mathrm{di}}$ given by TUCKER and GOULD ${ }^{5}$.

\section{The General Formula}

In connection with a study of the influence of atomic oxygen on the upper atmosphere, MASSEY and BATES ${ }^{23}$ were the first to consider, besides the radiative, the dielectronic recombination also, i. e. the inverse autoionization. In this process, an electron colliding with an ion is captured in a highly excited state of the ion. The surplus energy of the impact electron instead of being directly radiated, is used to excite another bound electron of the recombining ion. The latter, having been thus doubly excited, may undergo a transition by emitting a photon such that no autoionization is possible. In this way stabilization is achieved, the recombination takes place, and is accomplished dielectronically. The process can be represented as follows (BATES and Dalgarno ${ }^{24}$, Burgess ${ }^{1}$, TrefFtz ${ }^{20}$, Shore ${ }^{19}$ )

$$
\begin{aligned}
& \mathrm{X}^{+z}(i)+\mathrm{e}\left(E_{\mathrm{e}}, l_{\mathrm{e}}\right) \rightarrow \mathrm{X}^{+(z-1)}\left(i^{\prime}, i^{\prime \prime}\right), \\
& \mathrm{X}^{+(z-1)}\left(i^{\prime}, i^{\prime \prime}\right) \rightarrow \mathrm{X}^{+(z-1)}\left(i_{\mathrm{s}}, i^{\prime \prime}\right)+h v,
\end{aligned}
$$

where $\mathrm{X}^{+z}(i)$ is a $z$ times ionized atom in a state $i$, while $\mathrm{X}^{+(z-1)}\left(i^{\prime}, i^{\prime \prime}\right)$ is a $z-1$ times ionized atom in a doubly excited state $\left(i^{\prime}, i^{\prime \prime}\right) . E_{\mathrm{e}}, l_{\mathrm{e}}$ are the energy and angular momentum quantum number of the incident electron respectively; $v$ is the frequency of the emitted photon.

The states $i$ and $\left(i^{\prime}, i^{\prime \prime}\right)$ may in general be manyelectron states, differing obviously by one electron.

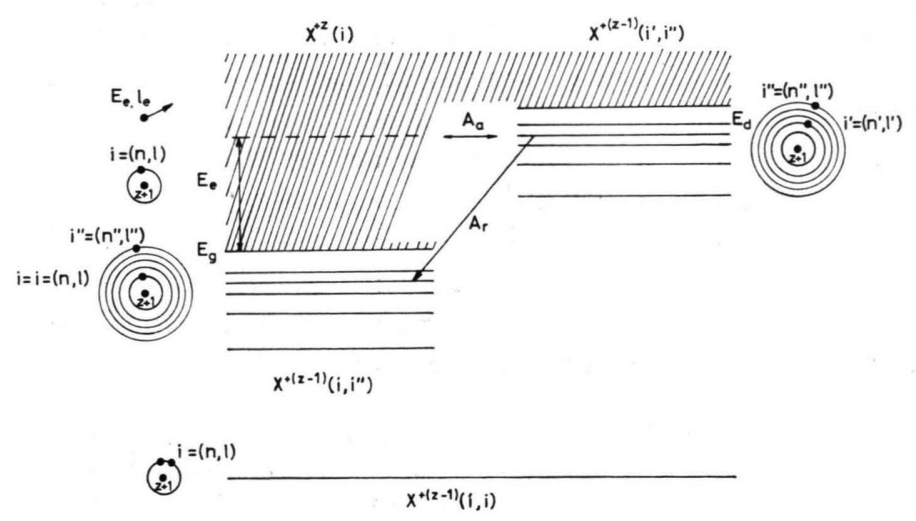

Fig. 1. Term scheme for dielectronic recombination.

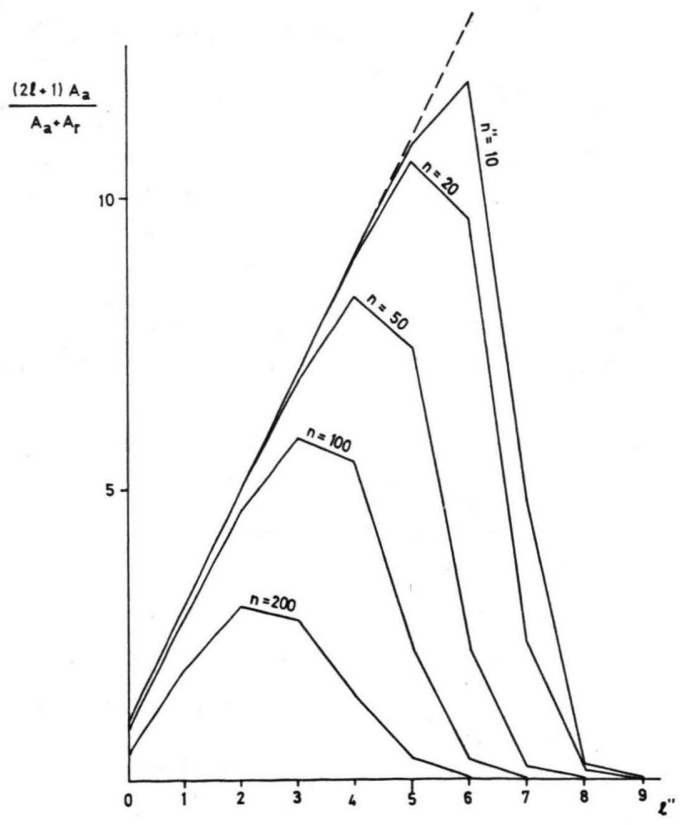

22 C. JoRdAN, M.N.R.A.S. 142, 499 [1969].

23 H. S. W. Massey and D. R. Bates, Rep. Prog. Phys. 9, 62 [1942/43].

24 D. R. BATES and A. Dalgarno, Electron Recombination, in: Atomic and Molecular Processes, Ed. D. R. BATEs, Academic Press, Inc., New York 1962, Chapter 7, esp. p. 246. 
Now, it can be shown ${ }^{25}$ that the most efficient recombination is obtained, if the state $i_{\mathrm{s}}$ of the inner electron of $\mathrm{X}^{+(z-1)}$ is configurationally the same as the state $i$ of the recombining ion $\mathrm{X}^{+z}$, the transition $i \rightarrow i^{\prime}$ being an optical one. In the following we shall therefore take $i=i_{\mathrm{s}}$ as granted. Moreover, if the states $i, i^{\prime}$, and $i^{\prime \prime}$ are characterized by their effective principal and angular momentum quantum numbers, viz.,

$$
i=(n, l), i^{\prime}=\left(n^{\prime}, l^{\prime}\right) \text { and } i^{\prime \prime}=\left(n^{\prime \prime}, l^{\prime \prime}\right)
$$

then the most important terms contributing to the process $P_{1}$ are those where

$$
l^{\prime}=l+1 \text { and } l^{\prime \prime}=l_{\mathrm{e}}-1 .
$$

The change in the energy of the incident electron used for excitation of the inner electron is given on one hand by

$$
E_{\mathrm{ex}}=E_{\mathrm{e}}+\frac{z^{2}}{n^{\prime \prime 2}} I_{\mathrm{H}}
$$

( $I_{\mathrm{H}}$ is the ionization energy of hydrogen), on the other by

$$
E_{\mathrm{ex}}=-\frac{(z+1)^{2}}{n^{\prime 2}} I_{\mathrm{H}}+\frac{(z+1)^{2}}{n^{2}} I_{\mathrm{H}}
$$

provided $n^{\prime \prime} \gg n^{\prime}$, i. e., we neglect the interaction between the captured and the excited electron, here. The assumption that the electrons are captured in highly excited states is in fact reasonable while considering the main contributions to $\alpha_{\mathrm{di}}$. The occupation of high levels makes also the dielectronic recombination quite important particularly in the case of radio recombination lines (GAYET et al. ${ }^{9}$, GolDBERG $^{10}$ ). Under this assumption $n^{\prime \prime} \gg n^{\prime}$ the energy of the highly excited states is

$$
E_{\mathrm{d}}=-\frac{(z+1)^{2}}{n^{\prime 2}} I_{\mathrm{H}}-\frac{z^{2}}{n^{\prime 2}} I_{\mathrm{H}},
$$

the ground state energy of the ion before recombination being

$$
E_{\mathbf{g}}=-\frac{I_{\mathrm{H}}(z+1)^{2}}{n^{2}} .
$$

Then, from (2.3) and (2.4)

$$
\begin{aligned}
E_{\mathrm{e}} & \equiv E=(z+1)^{2} I_{\mathrm{H}}\left(\frac{1}{n^{2}}-\frac{1}{n^{2}}\right)-\frac{z^{2}}{n^{\prime 2}} I_{\mathrm{H}} \\
& =E_{\mathrm{d}}-E_{\mathrm{g}}
\end{aligned}
$$

by (2.5) and (2.6), which can be seen from Fig. 1 .

One can now see immediately that the condition $i_{\mathrm{s}}=i$ is a sufficient condition for stabilization. The

25 See the paragraph following Eq. (2.7).

${ }^{26}$ To be more general, we now keep $i_{\mathrm{s}}$ different from $i$ till otherwise stated.

27 Here the state $i$ needs not be the ground state of the ion $\mathrm{X}+z$. energy of the stabilized state $b=\left(i_{\mathrm{s}}, i^{\prime \prime}\right)$ is namely

$$
E_{b}=-I_{\mathrm{H}} \frac{(z+1)^{2}}{n_{\mathrm{s}}^{2}}-I_{\mathrm{H}} \frac{z^{2}}{n^{\prime 2}} .
$$

For the stabilization of the recombination, it is clear that

that is

$$
E_{b}<E_{\mathrm{g}}=-\frac{(z+1)^{2}}{n^{2}} I_{\mathrm{H}},
$$

$$
-I_{\mathrm{H}} \frac{z^{2}}{n^{\prime \prime 2}}-I_{\mathrm{B}} \frac{(z+1)^{2}}{n_{\mathrm{s}}{ }^{2}}+I_{\mathrm{H}} \frac{(z+1)^{2}}{n^{2}}<0 .
$$

This inequality is in any way satisfied, if $n_{\mathrm{s}}=n$, i. e., $i_{\mathrm{s}}=i$. One can also see easily that in many cases $i_{\mathrm{s}}=i$ is also a necessary condition.

We denote the number densities of electrons by $N_{\mathrm{e}}$ and of the ions $\mathrm{X}^{+z}(i)$ by $N_{z}(i)$. Then the number of recombinations per $\mathrm{cm}^{3}$ per sec of ions in state $i$ is given by

$$
\begin{aligned}
\frac{\mathrm{d}}{\mathrm{d} t} N_{z}(i) & =\alpha_{\mathrm{di}}(i, \text { tot }) N_{\mathrm{e}} N_{z}(i), \\
& =\sum_{i^{\prime}, i^{\prime \prime}} \alpha_{\mathrm{di}}\left(i ; i^{\prime}, i^{\prime \prime}\right) N_{\mathrm{e}} N_{z}(i),
\end{aligned}
$$

where $\alpha_{\mathrm{di}}$ is the coefficient of dielectronic recombination.

On the other hand, the dielectronic recombination is ensured when the process $\mathrm{P}_{2}$ takes place ${ }^{26}$. Therefore, the total number of dielectronic recombinations is equal to the total number of stabilizations, namely 27

$$
\begin{gathered}
\sum_{i} \sum_{i^{\prime}, i^{\prime \prime}} \alpha_{\mathrm{di}}\left(i ; i^{\prime}, i^{\prime \prime}\right) N_{\mathrm{e}} N_{z}(i) \\
=\sum_{i^{\prime}, i^{\prime \prime}} N_{z-1}\left(i^{\prime}, i^{\prime \prime}\right) \sum_{i_{\mathrm{s}}} A_{\mathrm{r}}\left(i^{\prime}, i^{\prime \prime} \rightarrow i_{\mathrm{s}}, i^{\prime \prime}\right),
\end{gathered}
$$

where $N_{z-1}\left(i^{\prime}, i^{\prime \prime}\right)$ is the number density of the doubly excited ion $\mathrm{X}^{+(z-1)}$, and $A_{\mathrm{r}}$ is the transition probability for the process $\mathrm{P}_{2}$. Here $A_{\mathrm{r}}$ may be taken to be independent of the captured electron, i. e., the coupling between the inner (excited) and the outer (captured) electron is neglected. This approximation is borne out by the quantum mechanical treatment (SHORE ${ }^{19}$, TREFFTZ ${ }^{28}$ ), which will be discussed in the next paper.

Considering a stationary state, for every level the number of all electron losses due to photo- and autoionization are equal to the number of electron captures. Consequently, the number density $N_{z-1}$ can

28 E. Trefftz, in: Physics of the One- and Two-Electron Atoms, Ed. F. Bopp and H. Kleinpoppen, North Holland Publ. Comp., Amsterdam 1969. 
be determined from the steady-state condition:

$$
\begin{aligned}
& N_{z-1}\left(i^{\prime}, i^{\prime \prime}\right)\left[\sum_{i_{\mathrm{s}}} A_{\mathrm{r}}\left(i^{\prime}, i^{\prime \prime} \rightarrow i_{\mathrm{s}}, i^{\prime \prime}\right)\right. \\
& \left.\quad+\sum_{i^{\prime \prime \prime}} \sum_{l_{\mathrm{e}}} A_{\mathrm{a}}\left(i^{\prime}, i^{\prime \prime} \rightarrow i^{\prime \prime \prime}, E_{\mathrm{e}} l_{\mathrm{e}}\right)\right] \\
& =\sum_{i^{\prime \prime \prime}} N_{\mathrm{e}} N_{z}\left(i^{\prime \prime \prime}\right) \sum_{l_{\mathrm{e}}} r_{\text {cap }}\left(i^{\prime \prime \prime}, E_{\mathrm{e}} l_{\mathrm{e}} \rightarrow i^{\prime}, n^{\prime \prime} l^{\prime \prime}\right), \quad(2.11)
\end{aligned}
$$

where $A_{\mathrm{a}}$ is the probability of auto-ionization of the ion $\mathrm{X}^{+(z-1)}$ back to states $i^{\prime \prime \prime}$ of the ion $\mathrm{X}^{+(z)}$ and to the state $E_{\mathrm{e}}$ and $l_{\mathrm{e}}$ of the free electron. Here $r_{\text {cap }}$ denotes the rate of electron capture.

Substituting for $N_{z-1}$ from (2.11) in (2.10), one gets first

$$
\begin{aligned}
& \sum_{i} \sum_{i^{\prime}, i^{\prime \prime}} \alpha_{\mathrm{di}}\left(i ; i^{\prime}, i^{\prime \prime}\right) N_{z}(i) N_{\mathrm{e}} \\
& =\sum_{i^{\prime \prime \prime}} N_{z}\left(i^{\prime \prime \prime}\right) N_{\mathrm{e}} \sum_{i^{\prime}, i^{\prime \prime}} \frac{\sum_{l_{\mathrm{e}}} r_{\mathrm{cap}} \sum_{i_{\mathrm{s}}} A_{\mathrm{r}}}{\sum_{i_{\mathrm{s}}} A_{r}+\sum_{i^{\prime \prime}} \sum_{l_{\mathrm{e}}} A_{\mathrm{a}}} .
\end{aligned}
$$

In the following, we shall only be interested in the recombination to the ground state of $\mathrm{X}^{+z}$ which is denoted from now on by $i$. Equation (2.11') then gives

$$
\alpha_{\mathrm{di}}(i, \text { tot })=\sum_{i^{\prime}, i^{\prime \prime}} \frac{\sum_{l_{\mathrm{e}}} r_{\mathrm{cap}}\left(i, E_{\mathrm{e}} l_{\mathrm{e}} \rightarrow i^{\prime}, n^{\prime \prime} l^{\prime \prime}\right) \sum_{i_{\mathrm{s}}} A_{\mathrm{r}}}{\sum_{i_{\mathrm{s}}} A_{\mathrm{r}}+\sum_{i^{\prime \prime}} \sum_{l_{\mathrm{e}}} A_{\mathrm{a}}} .
$$

In order to set up a relation between $A_{\mathrm{a}}$ and $r_{\text {cap }}$, a thermodynamic equilibrium may now be assumed. Then the number of photons of frequency $v$ emitted due to process $\mathrm{P}_{2}$ should be equal to the number of photons of frequency $v$ absorbed. To maintain Maxwell distribution, the number of electrons of the energy $E$ lost due to capture must be gained by the auto-ionizaton. In other words, it means that the steady-state condition (2.11) holds true for every state of excitation $i^{\prime \prime \prime}$ of $\mathrm{X}^{+z}$ and in particular for the ground state $i$ without the first term on the left hand side. This can be stated for each value of the angular momentum quantum number $l_{\mathrm{e}}$ of the free electron, so that

$$
\begin{array}{r}
A_{\mathrm{a}}\left(i^{\prime}, i^{\prime \prime} \rightarrow i, E_{\mathrm{e}} l_{\mathrm{e}}\right) \\
=r_{\text {cap }}\left(i, E_{\mathrm{e}} l_{\mathrm{e}} \rightarrow i^{\prime}, i^{\prime \prime}\right)\left[\frac{N_{\mathrm{e}} N_{z}(i)}{N_{z-1}\left(i^{\prime}, i^{\prime \prime}\right)}\right],
\end{array}
$$

where the fraction in the square bracket can be evaluated with the aid of Saha and Boltzmann equations as given below.

First, the ratio of number densities $N_{z}$ and $N_{z-1}^{0}$ of the ions $\mathrm{X}^{+z}$ and $\mathrm{X}^{+(z-1)}$ in their ground state ${ }^{29}$

29 To distinguish $\mathrm{X}+(z-1)\left(i^{\prime}, i^{\prime \prime}\right)$ from $\mathrm{X}+(z-1)$ in ground state, we use a superscript zero in the number density of the latter. is given by the Saha ionization equation

$$
\frac{N_{z-1}^{0}}{N_{z} N_{\mathrm{e}}}=\frac{\omega_{z-1}^{0}}{\omega_{z}} \frac{h^{3}}{2(2 \pi m k T)^{3 / 2}} e^{E_{g} / k T}
$$

where $E_{\mathrm{g}}$ is the ionization energy for the ion $\mathrm{X}^{+z}$ and is given by $(2.6)$. Here $\omega_{z-1}^{0}$ and $\omega_{z}$ are the statistical weights of the respective ions.

Second, with the aid of Boltzmann equation the fraction of ions in the doubly excited state $\left(i^{\prime}, i^{\prime \prime}\right)$ with respect to the number $N_{z-1}$ in ground state can be expressed as

$$
\frac{N_{z-1}\left(i^{\prime}, i^{\prime \prime}\right)}{N_{z-1}^{0}}=\frac{\omega_{z-1}\left(i^{\prime}, i^{\prime \prime}\right)}{\omega^{0} z-1} e^{-E_{\mathrm{d}} / k T},
$$

$E_{\mathrm{d}}$ being given by (2.5). Eliminating $N^{0}{ }_{z-1}$ from $\left(2.13^{\prime}\right)$ with the help of (2.14) and using (2.7), we get finally

$$
\frac{N_{z-1}\left(i^{\prime}, i^{\prime \prime}\right)}{N_{z}(i) N_{\mathrm{e}}}=\frac{\omega\left(i^{\prime}, i^{\prime \prime}\right)}{\omega(i)} \frac{h^{3} e^{-E / k T}}{2(2 \pi m k T)^{3 / 2}},
$$

where we have omitted the subscripts of $\omega$ 's for the sake of conciseness. With (2.15), expression (2.13) goes into

$A_{\mathrm{a}}\left(i^{\prime}, i^{\prime \prime} \rightarrow i, E_{\mathrm{e}} l_{\mathrm{e}}\right)=r_{\text {cap }} \frac{2 \omega(i)}{\omega\left(i^{\prime}, i^{\prime \prime}\right)} \frac{(2 \pi m k T)^{3 / 2}}{h^{3}} e^{E / k T}$.

Substituting now from (2.16) for $r_{\text {cap }}$ in (2.12), one gets finally

$$
\begin{gathered}
\alpha_{\mathrm{di}}(i, \text { tot })=\frac{h^{3}}{2(2 \pi m k T)^{3 / 2}} \sum_{\substack{i^{\prime}=n^{\prime}, l^{\prime} \\
i^{\prime \prime}=n^{\prime \prime}, l^{\prime \prime}}} \frac{\omega\left(i^{\prime}, i^{\prime \prime}\right)}{\omega(i)} e^{-E / k T} \\
\times \frac{\sum_{i_{\mathrm{s}}} A_{\mathrm{r}}\left(i^{\prime}, i^{\prime \prime} \rightarrow i_{\mathrm{s}}, i^{\prime \prime}\right) \sum_{l_{\mathrm{e}}} A_{\mathrm{a}}\left(i^{\prime}, i^{\prime \prime} \rightarrow i, E_{\mathrm{e}} l_{\mathrm{e}}\right)}{\sum_{i_{\mathrm{s}}} A_{\mathrm{r}}\left(i^{\prime}, i^{\prime \prime} \rightarrow i_{\mathrm{s}}, i^{\prime \prime}\right)+\sum_{\substack{i^{\prime \prime} \\
i^{\prime \prime}, l_{\mathrm{e}}}} A_{\mathrm{a}}\left(i^{\prime}, i^{\prime \prime} \rightarrow i^{\prime \prime \prime}, E_{\mathrm{e}} l_{\mathrm{e}}\right)}(2.17)
\end{gathered}
$$

which is the most general formula. It may be mentioned here that exactly the same formula can be derived by the quantum theory of resonance scattering (TREFFTZ ${ }^{20}$, SHORE ${ }^{19}$ ), with the aid of which the quantities $A_{\mathrm{a}}, A_{\mathrm{r}}$ can also be calculated. Up to now, nothing has been said about the quantities $A_{\mathrm{r}}$, $A_{\text {a }}$ or $r_{\text {cap }}$. In the following sections we discuss however a few approximate formulas for $A_{\mathrm{a}}, A_{\mathrm{r}}$, and $\alpha_{\mathrm{di}}$ found in the literature.

\section{An Approximation for Low Temperature}

The first approximation of formula (2.17) which we would like to discuss is that of MASSEY and BATES $^{23}$. If one identifies the stable state with the ground state of $\mathrm{X}^{+z}(i)$, i. e., $i_{\mathrm{s}}=i$, and assumes that the auto-ionization takes place only to the continu- 
um of ion $\mathrm{X}^{+z}(i)$, i. e., $i^{\prime \prime \prime}=i$, Eq. (2.17) simplifies to

$$
\begin{gathered}
a_{\mathrm{di}}(i, \text { tot })=\frac{h^{3}}{2(2 \pi m k \mathrm{~T})^{3 / 2}} \sum_{i^{\prime}, i^{\prime \prime}} \frac{\omega\left(i^{\prime}, i^{\prime \prime}\right)}{\omega(i)} e^{-E / k T} \\
\times \frac{A_{\mathrm{r}}\left(i^{\prime}, i^{\prime \prime} \rightarrow i, i^{\prime \prime}\right) A_{\mathrm{a}}\left(i^{\prime}, i^{\prime \prime} \rightarrow i, E_{\mathrm{e}}\right)}{A_{\mathrm{r}}\left(i^{\prime}, i^{\prime \prime} \rightarrow i, i^{\prime \prime}\right)+A_{\mathrm{a}}\left(i^{\prime}, i^{\prime \prime} \rightarrow i, E_{\mathrm{e}}\right)},
\end{gathered}
$$

where

$$
A_{\mathrm{a}}\left(i^{\prime}, i^{\prime \prime} \rightarrow i, E_{\mathrm{e}}\right)=\sum_{l_{\mathrm{e}}} A_{\mathrm{a}}\left(i^{\prime}, i^{\prime \prime} \rightarrow i, E_{\mathrm{e}}, l_{\mathrm{e}}\right) .
$$

One may now introduce the lifetimes defined by

$$
\tau_{\mathrm{r}}=A_{\mathrm{r}}^{-1} \quad \text { and } \quad \tau_{\mathrm{a}}=A_{\mathrm{a}}^{-1},
$$

to get the formula

$\alpha_{\mathrm{di}}(i$, tot $)=\sum_{i^{\prime}, i^{\prime \prime}} \frac{1}{\tau_{\mathrm{a}}+\tau_{\mathrm{r}}} \frac{\omega\left(i^{\prime}, i^{\prime \prime}\right)}{2 \omega(i)} \frac{h^{3} e^{-E / k T}}{(2 \pi m k T)^{3 / 2}}$.

In cases where the lifetime $\tau_{\mathrm{r}}$ for emission of a line is much longer than the lifetime $\tau_{\mathrm{a}}$ for autoionization, i. e. $A_{\mathrm{a}} \gg A_{\mathrm{r}}$, Eq. (3.2) reduces to (MASSEY and BATES ${ }^{23}$, BATES and DALGARNo ${ }^{24}$ )

$$
\begin{array}{r}
\alpha_{\mathrm{di}}(i, \text { tot })=T^{-y / 2} \frac{\sqrt{2 \pi} e^{2} h}{m c^{3}(m k)^{3 / 2}} \sum_{i^{\prime}, i^{\prime \prime}} \frac{\omega\left(i^{\prime}, i^{\prime \prime}\right)}{\omega\left(i^{\prime}\right)} \\
\cdot\left(h v_{i^{\prime} i^{\prime}}\right)^{2} e^{-E / k T} f_{i^{\prime} i}
\end{array}
$$

where the lifetime $\tau_{\mathrm{r}}$ is expressed by the oscillator strength $f_{i i}$ :

$$
\tau_{\mathrm{r}}=A_{\mathrm{r}}^{-1}=\left[\frac{8 \pi^{2} e^{2} v^{2} \omega(i)}{m c^{3} \omega\left(i^{\prime}\right)} f_{i^{\prime} i}\right]^{-1} .
$$

For temperatures of the order of $1000-3000{ }^{\circ} \mathrm{K}$, (i. e. $k T \ll E$ ) the Boltzmann factor is quite small. Formula (3.3) is then applicable. In case of high density no upper levels will be formed. In the summation one has therefore only to take into account the lowest ones. Under these conditions BATES ${ }^{30}$ has shown, using formula (3.3), that $\alpha_{\mathrm{di}}$ is by a factor of 4-5 less than the corresponding radiative recombination coefficient $\alpha_{\mathrm{rad}}$ for $\mathrm{N}^{+}\left({ }^{3} \mathrm{P}\right)$ and is completely negligible for $\mathrm{O}^{+}\left({ }^{4} \mathrm{~S}\right)$. However, if the temperature is high and the density is low and if one substitutes $E$ from (2.7), it is obvious that the summation over $i^{\prime \prime}=\left(n^{\prime \prime}, l^{\prime \prime}\right)$ in (3.3) will diverge as

$$
\sum_{n^{\prime \prime}} n^{\prime \prime 2} e^{z^{2} / n^{\prime \prime 2} k T}
$$

where $n^{\prime \prime 2}$ comes from $\omega\left(n^{\prime}, l^{\prime} n^{\prime \prime} l^{\prime \prime}\right)$, see Eq. (7.2). This divergence disappears since for high temperature and for large $n^{\prime \prime}$ formula (3.3) is not applicable. The reason is that whereas $A_{\mathrm{r}}$ is constant with respect to $n^{\prime \prime}$ [see Eq. (7.3)], $A_{\mathrm{a}}$ decreases with

30 D. R. Bates, Planet. Space Sci. 9, 77 [1962]. increasing $n^{\prime \prime}$ [cf. the approximation of TUCKER and Gould ${ }^{5}$, Eq. (7.4) ]. Then for $A_{\mathrm{a}} \ll A_{\mathrm{r}}$ from the expression (3.1)

$$
\sum_{l^{\prime \prime}} \alpha_{\mathrm{di}}\left(i ; i^{\prime}, i^{\prime \prime}\right) \sim \sum_{l^{\prime \prime}} \omega\left(i^{\prime}, i^{\prime \prime}\right) A_{\mathrm{a}} \sim\left(n^{\prime \prime}\right)^{-3}
$$

(SHORE ${ }^{31}$ ) which makes the sum (3.2) converge. This rapid decrease of the summands can be substituted by a cut-off in (3.3). Before this cut-off becomes effective, large values of statistical weights $\omega\left(i^{\prime}, i^{\prime \prime}\right)$ may make the summands large which results in $\alpha_{\mathrm{di}}>\alpha_{\mathrm{rad}}$. In the following, we discuss a formula of $\alpha_{\mathrm{di}}$ for such cases.

\section{Burgess Simplified Formula for High Coronal Temperature}

To evaluate (2.17), naturally one needs to know the autoionization probability $A_{\mathrm{a}}$ as given by (2.16) or directly calculated quantum-mechanically $\left(\right.$ SHORE $\left.^{31}\right)$. However we follow Burgess ${ }^{1,2,12}$ here. Evidently, the rate of capture $r_{\text {cap }}$ can be found from the capture cross section $\sigma_{\text {cap }}$. For the capture into a highly excited state, $\sigma_{\text {cap }}$ may be approximated by the cross-section $\sigma_{\text {ex }}$ for ordinary excitation. Whereas in the case of excitation, the impact electron leaves the atom and therefore its energy remains positive, in the case of recombination the captured electron has a negative energy. By extrapolating $\sigma_{\mathrm{ex}}$ for negative energies of the captured electron just below threshold, the rate of capture $r_{\text {cap }}$ can be calculated as follows.

We consider the capture into closely lying highly excited states. The number of electrons captured into levels with the principal quantum numbers lying between $n^{\prime \prime}$ and $n^{\prime \prime}+\mathrm{d} n^{\prime \prime}$ per $N_{\mathrm{e}}$ and $N_{z}$ is given by $r_{\text {cap }} \mathrm{d} n^{\prime \prime}$. Here, the interval $\mathrm{d} n^{\prime \prime}$ corresponds to an energy interval $\mathrm{d} E$, which is populated by $\mathrm{d} f_{\mathrm{e}}$ recombining electrons; $f_{\mathrm{e}}$ being the Maxwell distribution function. From (2.7)

$$
\mathrm{d} E=I_{\mathrm{H}} \frac{2 z^{2}}{n^{\prime \prime 3}} \mathrm{~d} n^{\prime \prime} .
$$

The number of captures is, on the other hand, given by $\sigma_{\text {cap }} v_{\mathrm{e}} \mathrm{d} f_{\mathrm{e}}$, where $v_{\mathrm{e}}$ is the electron velocity. Hence

$$
\begin{aligned}
r_{\text {cap }} \mathrm{d} n^{\prime \prime} & =\sigma_{\text {cap }} v_{\mathrm{e}} \mathrm{d} f_{\mathrm{e}} \\
r_{\text {cap }} & =\sigma_{\text {cap }} \frac{4 \sqrt{2}}{\sqrt{\pi m}} \frac{z^{2} I_{\mathrm{H}}}{n^{\prime \prime 3}} \frac{E e^{-E / k T}}{(k T)^{3 / 2}} .
\end{aligned}
$$

31 B. W. Shore, Scientific Report No. 23, Harvard College Observatory, Cambridge 1969. 
With this, Eq. (2.16) takes the form

$$
\begin{aligned}
& A_{\mathrm{a}}\left(n^{\prime} l^{\prime}, n^{\prime \prime} l^{\prime \prime} \rightarrow n l, E_{\mathrm{e}} l_{\mathrm{e}}\right) \\
& \quad=\frac{32 \pi m}{h^{3}} \frac{\omega(n l)}{\omega\left(n^{\prime} l^{\prime}, n^{\prime \prime} l^{\prime \prime}\right)} \frac{z^{2} I_{\mathrm{H}}}{n^{\prime \prime 3}} E \sigma_{\text {cap }} .
\end{aligned}
$$

As mentioned above, $\sigma_{\text {cap }}$ is approximated by Burgess with the aid of $\sigma_{\text {ex }}$. Substitution of (4.4) in (2.17) leads to the following formula which is the same as the first Burgess formula ${ }^{31 a}$ (BURGESs ${ }^{32}$ ).

$$
\alpha_{\mathrm{di}}=T^{-3 / 2} \sum_{n^{\prime} l^{\prime}} \frac{C A_{\mathrm{r}} E e^{-E / k T n^{\prime \prime}-3} \sum_{l_{\mathrm{e}}} \sigma_{\mathrm{ex}}}{\sum_{n l} A_{\mathrm{r}}+\sum_{n^{\prime \prime} l^{\prime \prime} l_{\mathrm{e}}} C C^{\prime} \frac{\omega\left(n^{\prime \prime \prime} l^{\prime \prime \prime}\right)}{\omega\left(n^{\prime} l^{\prime}, n^{\prime \prime} l^{\prime \prime}\right)} \sigma_{\mathrm{ex}} E n^{\prime \prime-3}},
$$

where $\quad C=4 \sqrt{2} \mathrm{z}^{2} I_{\mathrm{H}} k^{-3 / 2}(\pi m)^{-1 / 2}$

and $\quad C^{\prime}=32 \pi m z^{2} I_{\mathrm{H}} h^{-3}$.

In most of the calculated cases the sums in the denominator reduce to one term each. Now a rough study of $\left(2 l^{\prime \prime}+1\right) \frac{A_{\mathrm{a}}}{A_{\mathrm{r}}+A_{\mathrm{a}}}$ as a function of $n^{\prime \prime} l^{\prime \prime}$ for the recombination process $\mathrm{Ca}^{+}+\mathrm{e} \rightarrow \mathrm{Ca}(i=4 \mathrm{~s}$, $i^{\prime}=4 \mathrm{p}$; BURGESs ${ }^{32}$ ) as well as a direct quantummechanical calculation (SHORE ${ }^{32 a}$ ) of the expression

$$
B\left(i^{\prime}, n^{\prime \prime}\right)=\sum_{l^{\prime \prime}} \frac{\omega\left(l^{\prime \prime}\right) \sum_{l_{\mathrm{e}}} A_{\mathrm{a}}}{\sum_{i_{\mathrm{s}}} A_{\mathrm{r}}+\sum_{i^{\prime \prime} l_{\mathrm{e}}} A_{\mathrm{a}}}
$$

show that quite large values of $n^{\prime \prime}$ actually contribute predominantly to $\alpha_{\mathrm{di}}$ [Figs. 2 (see p. 1782)

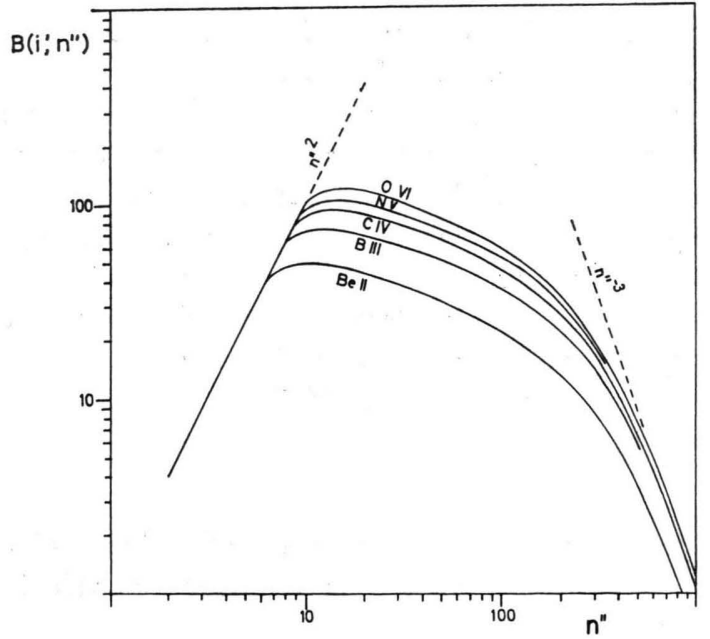

Fig. 3. Values of $B\left(i, n^{\prime \prime}\right)$ for the Be II-Sequence $\left(i=1 \mathrm{~s}^{2} 2 \mathrm{~s}\right.$, $i^{\prime}=1 \mathrm{~s}^{2} 2 \mathrm{p}$ ) (reproduced from SHORE ${ }^{19}$ ). 31. The stabilization is considered again only to the ground
state $i$ of the ion $\mathrm{X}+z(i)$. and 3]. This very fact allows the derivation of a simple formula for $\alpha_{\mathrm{di}}$.

In (4.5), the kinetic energy of the impact electron is given by $(2.7)$. Introducing the notation

$$
\varepsilon_{i i^{\prime}}=\left(1 / n^{2}-1 / n^{\prime 2}\right)
$$

where the effective principal quantum number $n$ is given by

$$
n=(z+1) \sqrt{R / T_{n}},
$$

where $R$ is the Rydberg constant and $T_{n}$ the term value in $\mathrm{cm}^{-1}$, one may rewrite the exponent of the function in (4.5) as

$E / k T=0.158 t^{-1}(z+1)^{2} \varepsilon_{i i^{\prime}}\left(1-\frac{z^{2}}{(z+1)^{2} \varepsilon_{i i^{\prime}} n^{\prime \prime 2}}\right)$.

Here

$$
t=10^{-6} T^{\circ} \mathrm{K}
$$

is the temperature in units of $10^{6}{ }^{\circ} \mathrm{K}$.

So far as the summation over $n^{\prime \prime}$ is concerned, it is clear that due to the factor in the parenthesis in (4.7), $E / k T$ behaves as a slowly varying function for the large values of $n^{\prime \prime}$ in which one is actually interested. As a result of this, one may replace $E / k T$ by a constant effective value $\tilde{E} / k T$, where (BuRgess ${ }^{12}$ )

$\tilde{E} / k T=0.158 t^{-1}(z+1)^{2} \varepsilon_{i i^{\prime}}\left[1+0.015 \frac{z^{3}}{(z+1)^{2}}\right]^{-1}$
$(\tilde{E} / k T \leqq 5.0)$

Now one can extract the exponential function from the $\operatorname{sum} \sum_{n^{\prime \prime} l^{\prime \prime}}$ in (4.5) and need not evaluate it for each $n^{\prime \prime}$. The summation over $n^{\prime \prime} l^{\prime \prime}$ can then be performed numerically. Expressing $A_{\mathrm{r}}$ in terms of the oscillator strength, BURGESs ${ }^{2}$ represents the results by the following formula

$$
\begin{aligned}
\alpha_{\mathrm{di}}=3.33 \cdot 10^{-13} t^{-3 / 2}(z+1)^{3} B(z) \\
\cdot \sum_{i^{\prime}} \sqrt{\varepsilon_{i i^{\prime}}} f_{i i^{\prime}} A(x) e^{-\tilde{E} / k T} \mathrm{~cm}^{3} \mathrm{sec}^{-1}
\end{aligned}
$$

where

$$
x=(z+1) \varepsilon_{i i^{\prime}}
$$

and only one summation over the bound states $i^{\prime}$ of the recombining ion $\mathrm{X}^{+z}$ remains to be carried out in each case. The functions $A(x)$ and $B(z)$ are tabulated below (Burgess ${ }^{2}$ ). The approximations (4.8) and (4.9) are based on a large number of numerical calculations.

\footnotetext{
32 A. Burgess, Proc. Second Harvard-Smithsonian Conf. on Stellar Atm., Smithsonian Astrophys. Obs., Special Report No. 174, 47 [1965].

32a B. W. SHORE, Astrophys. J. 158, 1205 [1969].
} 
A somewhat different form can be given to the formula (4.9), if one uses a polynomial representation of the functions $A(x)$ and $B(z)$. One has (BURGESS ${ }^{11}$ )

$\alpha_{\mathrm{di}}=3.0 \cdot 10^{-12} t^{-3 / 2} B^{\prime}(z) \sum_{i^{\prime}} f_{i i^{\prime}} A^{\prime}(x) e^{-\tilde{E} / k T}$

where now

$$
B^{\prime}(z)=z^{1 / 2}(z+1)^{5 / 2}\left(z^{2}+13.4\right)^{-1 / 2}, z \leqq 20
$$

and

$A^{\prime}(x)=x^{1 / 2}\left[1+0.105 x+0.015 x^{2}\right]^{-1}, x>0.05$.

Formula (4.9) along with Table 1 has been used for calculating $\alpha_{\mathrm{di}}$ for various ions (MüCKLICH ${ }^{16,33}$ ). We shall return to these calculations in the following sections. Burgess' detailed calculations of specific ions with the aid of the first formula (4.5), show that formula (4.9) or (4.11) give in general results within $20 \%$ of accuracy.

\section{Determination of the Oscillator Strengths}

In order to evaluate the simplified formula (4.9) numerically, one has to know first of all the oscillator strengths $f_{i^{\prime} i}$. In Coulomb approximation (BATES and DAMGAARD ${ }^{35}$ ), it is given by

$$
f_{i^{\prime} i}=3.037 \cdot 10^{-6} \tilde{\nu} \frac{S(\mathcal{M})}{\omega(i)} \sigma_{\text {B.D. }}^{2} .
$$

where $\tilde{\boldsymbol{v}}$ is the wave number of the transition in $\mathrm{cm}^{-1}, \sigma_{\mathrm{B} . \mathrm{D}}$. is the Coulomb integral (tabulated by Bates and Damgaard) and $S(\mathcal{M})$ is the relative multiplet strength which can be expressed as a pro- duct of Racah and fractional parentage coefficients (RoHRLICH ${ }^{36}$ ). The Coulomb integral is

$$
\sigma_{\text {B.D. }}=\frac{1}{z+1} \mathcal{F}\left(n_{1}, 1\right) \mathcal{J}\left(n_{1-1}, n_{1}, \mathrm{l}\right)
$$

where the functions $\mathcal{F}$ and $\mathcal{J}$ have been tabulated by BATES and DAMGAARD ${ }^{35}$, and $n_{1}$ is the effective principal quantum number, given by $\left(4.6^{\prime}\right)$.

For simplicity we have used the Coulomb approximation throughout to calculate oscillator strengths $f_{i^{\prime} i}$. For some ions of interest the oscillator strengths are enlisted in WIESE's ${ }^{37}$ tables. Some of these $f_{i^{\prime} i}$ are also calculated by Bates and Damgaard's method. A comparison of our calculated $f_{i^{\prime} i}$ with those of Wiese shows in all cases an agreement within the uncertainty of Bates and Damgaard's approximation - sometimes even a very good agreement.

The main task of the calculation is to determine the effective principal quantum number $n^{\prime}$ given by $\left(4 \cdot 6^{\prime}\right)$. As the term values are not always known for all ions of interest, one is obliged to take recourse to extrapolation. Since $n^{\prime}$ increases nearly monotonically within an isoelectronic sequence, it was estimated for these ions by extrapolating the corresponding values of $n^{\prime}$ of those members of the isoelectronic sequence for which the term values of the state in question are known. The corresponding term values can then be estimated using formula $\left(4.6^{\prime}\right)$. For ions, comprising a number of equivalent electrons in their outermost shell, the difference between the various term values in a transition array is small. In these cases mean values of $n^{\prime}$ were determined with the aid of mean term values over a transition array.

\begin{tabular}{llllllllllll}
\hline Argument & 0 & 1 & 2 & 3 & 4 & 5 & 6 & 7 & 8 & 9 & 10 \\
$A(x)[x>0.05]$ & 1.13 & 1.00 & 0.88 & 0.77 & 0.67 & 0.58 & 0.51 & 0.44 & 0.39 & 0.34 & 0.31 \\
$B(z)$ & 2.07 & 2.80 & 2.99 & 3.00 & 3.93 & 2.82 & 2.70 & 2.60 & 2.60 & 2.50 & 2.42 \\
Argument & 11 & 12 & 13 & 14 & 15 & 16 & 17 & 18 & 19 & 20 & 21 \\
$A(x)$ & 0.28 & 0.255 & $0.23_{5}$ & 0.21 & 0.19 & $0.17_{5}$ & 0.16 & $0.14_{5}$ & 0.13 & 0.12 & 0.11 \\
$B(z)$ & 2.34 & 2.26 & 2.18 & 2.11 & 2.05 & 1.98 & 1.92 & 1.88 & 1.82 & 1.78 & \\
\hline
\end{tabular}

Table 1.

33 Mücklich's result had already been used for determining the state of ionization which in turn was employed in the theory of line spectrum for the solar X-rays (ELWERT and MÜCKLICH ${ }^{34}$, ELWERT ${ }^{6}$ ). The theory agrees fairly with the observations.

34 G. Elwert and P. Mücklich, Mitt. d. Astron. Ges., Hamburg, p. 87, Reports of the Meeting in Eisenach/Thür., 6. -11. September 1965.
35 D. R. Bates and A. Damgand, Phil. Trans. Roy. Soc. London A 242, 101 [1949/50].

36 F. Rohrlich, Astrophys. J. 121, 441, 449 [1959].

37 W. L. Wiese, W. M. Smith, and B. M. Glennon, Atomic Transition Probabilities, Vol. 1, NSRDS-NBSA, U.S. Dept. of Commerce, Washinton D.C. 1966.

38 C. W. Allen, Astrophysical Quantities, Univ. of London, The Athlone Press, Second Edition, p. 54, 1963. 
The existing tables (ALLEN ${ }^{38}$, SHORE ${ }^{19}$ ) of the relative multiplet strengths were found to be insufficient. They were therefore calculated for the transitions in question, which in turn are mostly of the type $l^{n} \rightarrow l^{n-1} l^{\prime}$ or $l^{n} l^{\prime m-1} \rightarrow l^{n-1} l^{\prime m}$ in the notation of RoHRLich ${ }^{36}$. For the special case

$$
p^{n}(S L) \rightarrow p^{n-1}\left(S_{1} L_{1}\right) \mathrm{d} S L^{\prime},
$$

the Menzel and Goldberg ${ }^{39}$ relation gives

$$
\sum_{S_{1} L_{1}} \sum_{L^{\prime}} S(\mathcal{M})=10 n(2 S+1)(2 L+1) \text {. }
$$

The sum over the whole transition array is then

$$
\sum_{S L} \sum_{S_{1} L_{1}} \sum_{L^{\prime}} S=10 n\left(\begin{array}{l}
6 \\
n
\end{array}\right) \text {. }
$$

The relative multiplet strength for the individual transitions can be taken from the Table 2 .

\section{Results}

In the following section we discuss the results of the calculations of $\alpha_{\mathrm{di}}$ for the ions $\mathrm{C} \mathrm{V}$ and C VI; $\mathrm{N}$ VI and N VII; O VII and O VIII; Ne VII, Ne VIII and $\mathrm{Ne} \mathrm{IX;} \mathrm{Mg}$ IX, $\mathrm{MgX}$ and $\mathrm{MgXI}$; Si VI to SiXII; S VIII to SXIII and FeX to FeXIV (MÜCKLICH ${ }^{16}$, ANSARI et al. ${ }^{15}$, ElWERT ${ }^{17}$ ).

\begin{tabular}{cccccccc}
\hline & \multicolumn{6}{c}{$S(\mathscr{M})$} \\
$L$ & $L_{1}$ & $L^{\prime}$ & $n=$ & number of equivalent electrons \\
& & & 1 & 2 & 3 & 4 & 5 \\
\hline$S$ & $P$ & $P$ & - & 20 & 120 & 40 & - \\
$P$ & $S$ & $D$ & 60 & - & 40 & 120 & 20 \\
$P$ & $P$ & $P$ & - & 45 & 22.5 & 22.5 & 45 \\
$P$ & $P$ & $D$ & - & 135 & 67.5 & 67.5 & 135 \\
$P$ & $D$ & $S$ & - & - & 10 & 30 & 20 \\
$P$ & $D$ & $P$ & - & - & 22.5 & 67.5 & 45 \\
$P$ & $D$ & $D$ & - & - & 17.5 & 52.5 & 35 \\
$D$ & $P$ & $P$ & - & 1 & 1.5 & 0.5 & - \\
$D$ & $P$ & $D$ & - & 15 & 22.5 & 7.5 & - \\
$D$ & $P$ & $F$ & - & 84 & 126 & 42 & - \\
$D$ & $D$ & $P$ & - & - & 13.5 & 13.5 & - \\
$D$ & $D$ & $D$ & - & - & 52.5 & 52.5 & - \\
$D$ & $D$ & $F$ & - & - & 84 & 84 & - \\
\hline
\end{tabular}

Table 2. Relative multiplet strength for the transition $p^{n} S L \rightarrow p^{n-1}\left(S_{1} L_{1}\right) d S L^{\prime}$

With regards to numerical computations we will give formulas for $\alpha_{\mathrm{di}}$ instead of tables. According to the expression of Burgess the coefficient of dielectronic recombination can generally be written in the form

$$
\alpha_{\mathrm{di}}(i, \text { tot })=t^{-\mathrm{s} / 2} \sum_{i^{\prime}} F_{i^{\prime}} \exp \left\{-P_{i^{\prime}} / t\right\}
$$

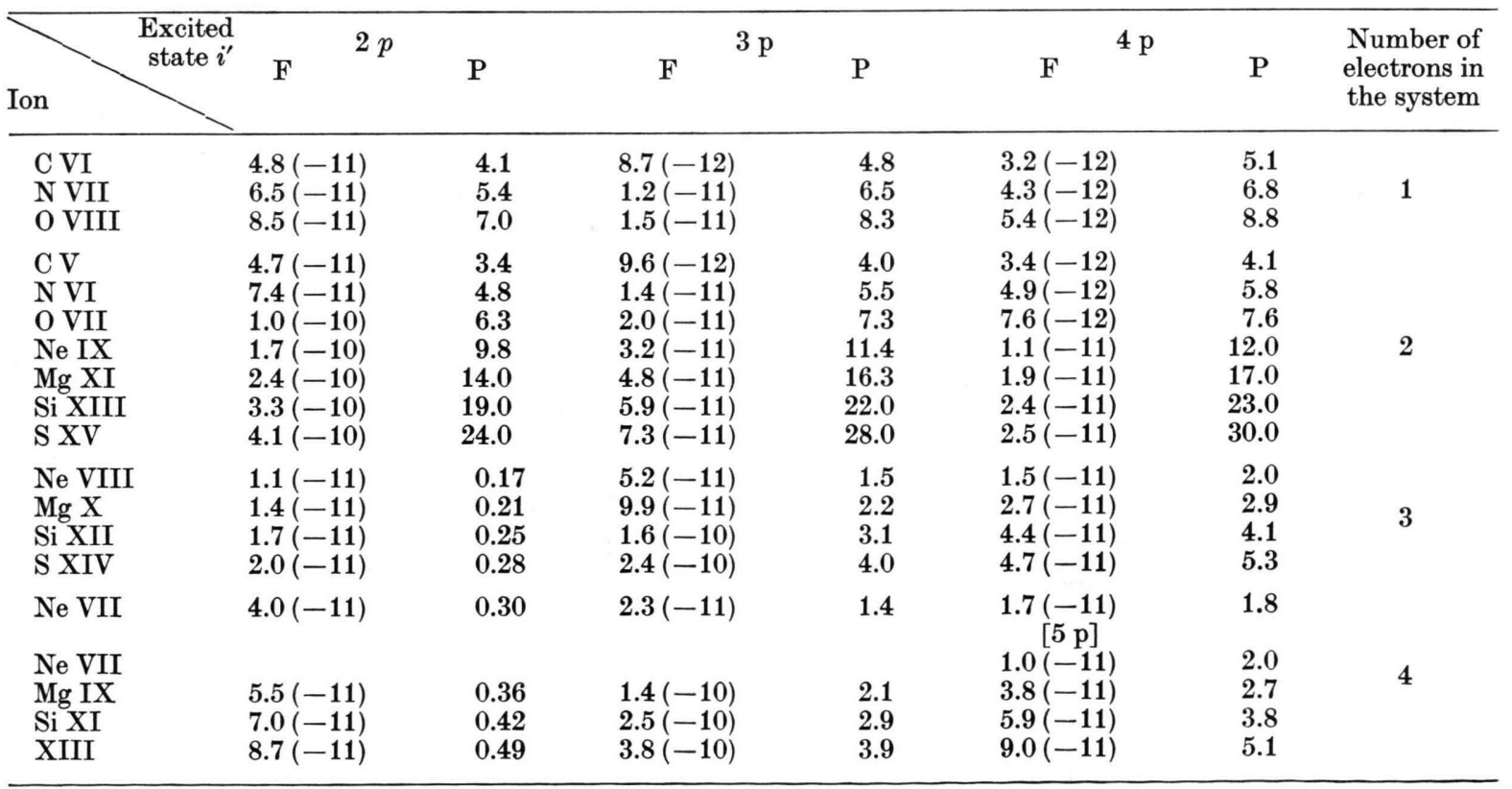

Table 3 .

39 D. H. Menzel and L. Goldberg, Astrophys. J. 84, 1 [1936]. 
where the parameters $F_{i^{\prime}}$ and $P_{i^{\prime}}$ take certain values for each ion. As a consequence of the fast decrease of the terms of the sum with increasing quantum numbers $i^{\prime}$, one has to consider only a few transitions. The parameters $F_{i^{\prime}}$ and $P_{i^{\prime}}$ are represented in Tables 3 and 4.

The results are represented in Figs. 4 to 13. One sees immediately that $\alpha_{\mathrm{di}}$ increases first with increasing temperature; the physical reason being that at high temperature more free electrons exist wich have the necessary energy to recombine via inverse autoionization followed by radiative stabilization. Then, $\alpha_{\mathrm{di}}$ reaches a maximum and decreases monotonically with increasing temperature. According to AlleN ${ }^{40}$ the higher the energy of the first optically allowed transition of the recombining ion, the higher is the temperature $T_{\max }$ at which the maximum of the dielectronic recombination occurs. $T_{\max }$ is approximately given by (ALLEN ${ }^{40}$ )

$$
T_{\max } \approx 90 \cdot \frac{10^{6}}{\hat{\lambda}_{[\mathrm{A}]}} \approx 7000 E[\mathrm{eV}]{ }^{\circ} \mathrm{K}
$$

where $\lambda$ is the wavelength of the first optically allowed transition of the recombining ion in $\AA$, and
$E$ is the corresponding excitation potential in $\mathrm{eV}$. On the other hand, the coefficient for radiative recombination $\alpha_{\mathrm{rad}}$ decreases monotonicaly with increasing temperature. As a result of this, the coefficients of dielectronic and radiative recombination behave differently depending on the temperature. A sufficiently good formula for $\alpha_{\text {rad }}$ was first derived by ELWERT ${ }^{41}$. In the figures the simpler expression given by BURGESS and SEATON ${ }^{3}$

$$
\alpha_{\mathrm{rad}}\left(\mathrm{X}^{+(z-1)}\right)=1.3 \cdot 10^{-15} \frac{z^{2}}{t} I_{z-1}^{1 / 2} \mathrm{~cm}^{3} \mathrm{sec}^{-1}
$$

was used. The ionization potential in $\mathrm{eV}$ of $\mathrm{X}^{+(z-1)}$ is denoted by $I_{z-1}$. In Table $5, T_{\max }$ for the ions listed above is given. The temperature $T_{\min }$ at which the total recombination coefficient $\alpha_{\mathrm{di}}+\alpha_{\mathrm{rad}}$ becomes a minimum is according to ALLEN ${ }^{40}$ given by

$$
T_{\min } \approx 0.25 T_{\max } \text {. }
$$

The following interesting point should be noted. In case of $\mathrm{C} \mathrm{V}$ and $\mathrm{C} \mathrm{VI,} \mathrm{Fig.} \mathrm{4,} \mathrm{the} \mathrm{maximum} \mathrm{of}$ $\alpha_{\mathrm{di}}$ occurs at about $2 \cdot 10^{6}{ }^{\circ} \mathrm{K}$, the first excitation

\begin{tabular}{|c|c|c|c|c|c|c|c|c|c|c|c|}
\hline \multirow{2}{*}{ Ion $i^{\prime}$} & \multicolumn{2}{|l|}{$3 \mathrm{~d}$} & \multicolumn{2}{|l|}{$4 \mathrm{~d}$} & \multicolumn{2}{|l|}{$5 \mathrm{~d}$} & \multicolumn{2}{|l|}{$3 \mathrm{~s}$} & \multicolumn{2}{|l|}{$\mathrm{p}^{n}$} & \multirow{2}{*}{$\begin{array}{l}\text { Number } \\
\text { of electr } \\
\text { in the } \\
\text { system }\end{array}$} \\
\hline & F & $\mathbf{P}$ & F & $\mathbf{P}$ & $\mathrm{F}$ & $\mathbf{P}$ & $\mathbf{F}$ & $\mathbf{P}$ & $F^{r}$ & $\mathbf{P}$ & \\
\hline Si X & $1.7(-10)$ & 2.6 & $4.0(-11)$ & 3.3 & $1.7(-11)$ & 3.6 & $6.9(-12)$ & 2.4 & $6.8(-11)$ & 0.46 & 5 \\
\hline S XII & $2.8(-10)$ & 3.5 & $6.2(-11)$ & 4.5 & $2.5(-11)$ & 4.9 & $8.2(-12)$ & 3.3 & $9.0(-11)$ & 0.56 & 5 \\
\hline Si IX & $2.4(-10)$ & 2.4 & $6.4(-11)$ & 2.9 & - & - & $9.8(-12)$ & 2.1 & $5.2(-11)$ & 0.49 & 6 \\
\hline S XI & $4.0(-10)$ & 3.3 & $1.0(-10)$ & 4.1 & $3.9(-11)$ & 4.6 & $1.5(-11)$ & 3.0 & $7.0(-11)$ & 0.61 & 6 \\
\hline Si VIII & $1.7(-10)$ & 2.1 & $5.9(-11)$ & 2.6 & - & - & $9.3(-12)$ & 1.9 & $3.8(-11)$ & 0.52 & 7 \\
\hline S X & $4.5(-10)$ & 3.0 & $1.1(-10)$ & 3.7 & $4.4(-11)$ & 4.0 & $2.0(-11)$ & 2.6 & $5.2(-11)$ & 0.61 & 7 \\
\hline Si VII & $7.5(-11)$ & 1.9 & $1.3(-11)$ & 2.3 & - & - & $4.1(-12)$ & 1.6 & $2.5(-11)$ & 0.54 & 8 \\
\hline S IX & $2.5(-10)$ & 2.7 & $1.1(-10)$ & 3.3 & $3.0(-11)$ & 3.4 & $1.0(-11)$ & 2.4 & $3.4(-11)$ & 0.64 & 8 \\
\hline Si VI & $6.9(-11)$ & 1.6 & $1.8(-11)$ & 1.9 & $8.2(-12)$ & 2.1 & $6.7(-12)$ & 1.4 & $1.0(-11)$ & 0.56 & 9 \\
\hline S VIII & $2.6(-10)$ & 2.5 & $9.8(-11)$ & 2.9 & $2.5(-11)$ & 3.2 & $1.5(-11)$ & 2.1 & $1.5(-11)$ & 0.67 & 9 \\
\hline Fe XVII & $2.7(-9)$ & 7.8 & $7.2(-10)$ & 9.7 & - & - & $1.0(-10)$ & 7.0 & $3.6(-11)$ & 8.59 & 10 \\
\hline
\end{tabular}
energies of both ions being of the same order of

\begin{tabular}{|c|c|c|c|c|c|c|c|c|c|c|c|}
\hline Ion & $\mathrm{F}^{3 \mathrm{p}}$ & $\mathrm{P}$ & $\mathrm{F}^{4 \mathrm{p}}$ & $\mathrm{P}$ & $\mathrm{F}^{5 \mathrm{p}}$ & $\mathbf{P}$ & & & & & \\
\hline $\mathrm{Fe}$ XVI & $1.3(-10)$ & 0.3 & $1.8(-10)$ & 2.4 & $6.3(-11)$ & 3.3 & - & - & & - & 11 \\
\hline Fe XV & $3.6(-10)$ & 0.5 & $2.9(-10)$ & 2.3 & $1.2(-12)$ & 3.3 & - & - & - & - & 12 \\
\hline Ion & $F^{3 \mathrm{~d}}$ & $P$ & $F^{4 d}$ & $P$ & $\mathrm{~F}^{5 \mathrm{~d}}$ & $\mathbf{P}$ & $4 \mathrm{~s}$ & $P$ & $\mathrm{p}^{n}$ & P & \\
\hline Fe XIV & $9.1(-11)$ & 0.5 & $1.4(-10)$ & 2.1 & $4.9(-11)$ & 2.8 & $2.9(-11)$ & 1.8 & $2.2(-10)$ & 0.42 & 13 \\
\hline Fe XIII & $9.0(-11)$ & 0.4 & $3.4(-10)$ & 1.8 & $1.1(-10)$ & 2.5 & $2.3(-11)$ & 1.6 & $1.5(-10)$ & 0.37 & 14 \\
\hline Fe XII & $9.2(-11)$ & 0.3 & $4.5(-10)$ & 1.7 & $1.4(-10)$ & 2.3 & $4.8(-11)$ & 1.6 & $8.6(-11)$ & 0.33 & 15 \\
\hline $\mathrm{Fe} \mathrm{XI}$ & $6.2(-11)$ & 0.3 & $5.9(-10)$ & 1.6 & $1.7(-10)$ & 2.1 & $4.2(-11)$ & 1.4 & $3.3(-11)$ & 0.25 & 16 \\
\hline $\mathrm{Fe} \mathrm{X}$ & $6.4(-11)$ & 0.3 & $6.1(-10)$ & 1.5 & $1.7(-10)$ & 2.0 & $4.1(-11)$ & 1.4 & $1.8(-11)$ & 0.30 & 17 \\
\hline
\end{tabular}
magnitude. On the other hand, if the first optically

Table 4. 

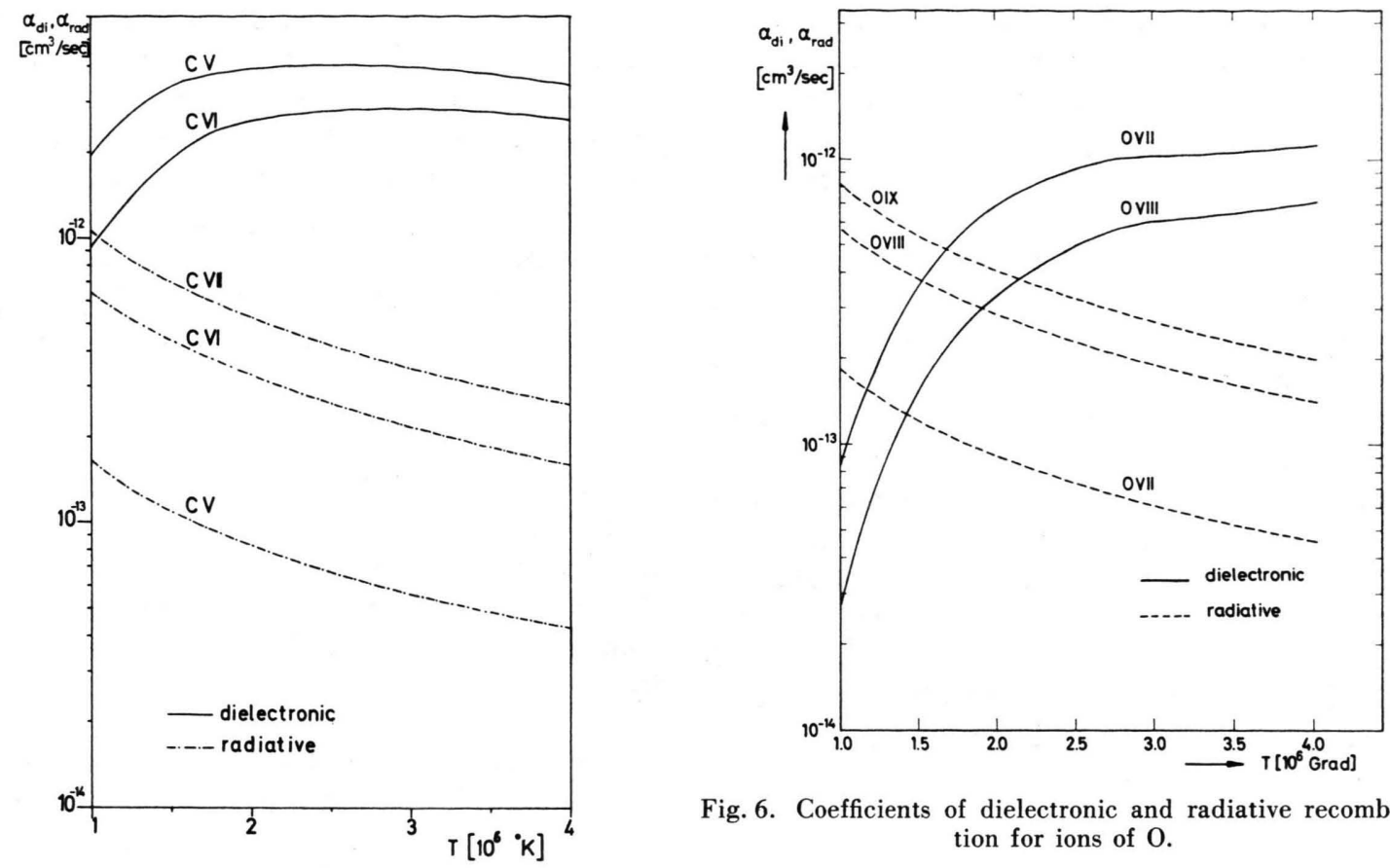

Fig. 6. Coefficients of dielectronic and radiative recombination for ions of $\mathbf{O}$.

Fig. 4. Coefficients of dielectronic and radiative recombination for ions of $\mathrm{C}$.

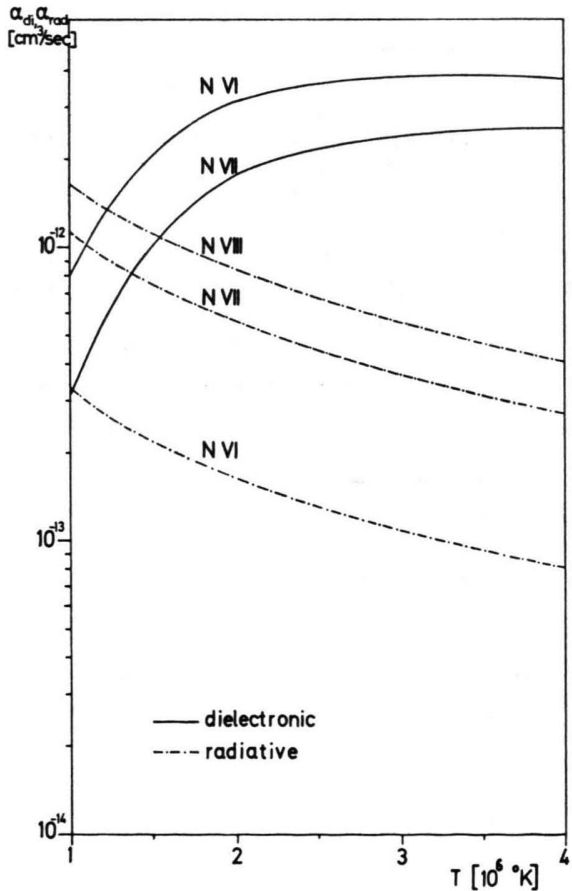

Fig. 5. Coefficients of dielectronic and radiative recombina. tion for ions of $\mathrm{N}$.

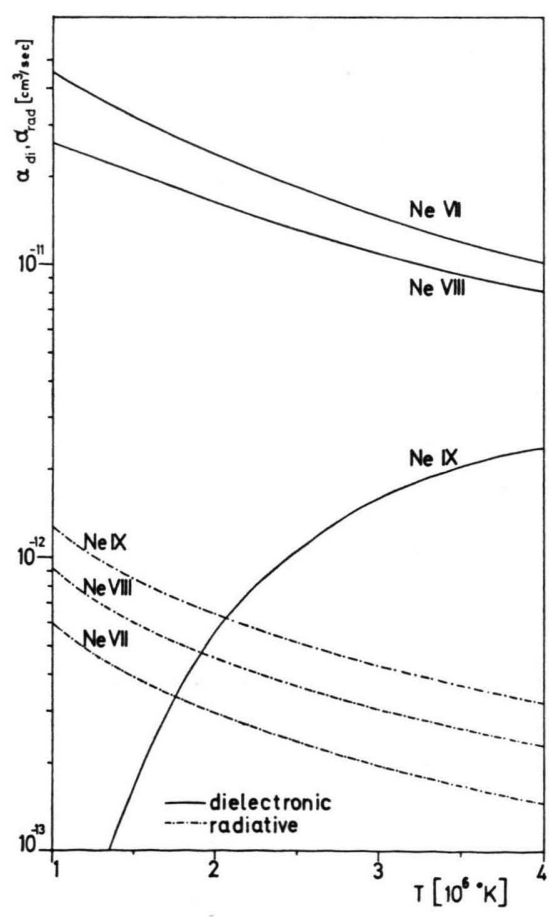

Fig. 7. Coefficients of dielectronic and radiative recombination for ions of Ne. 


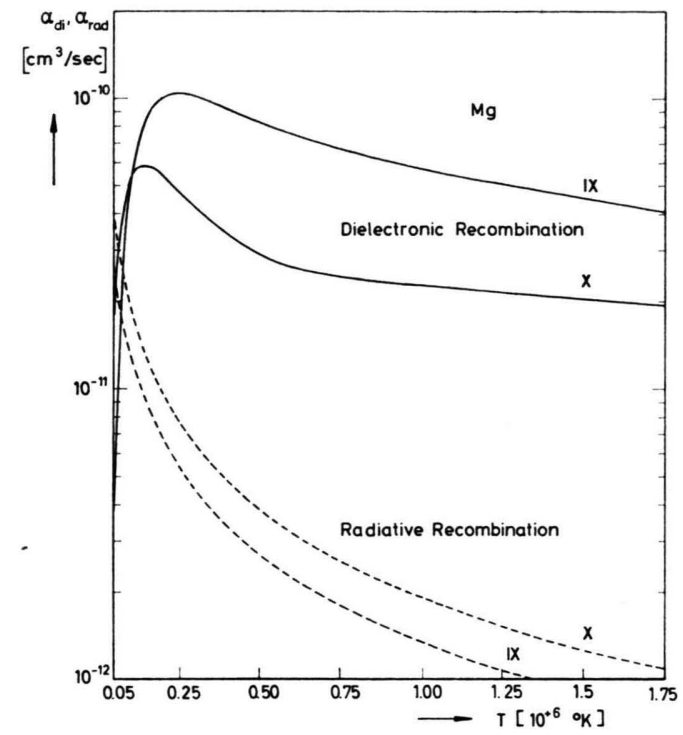

Fig. 8. Coefficients of dielectronic and radiative recombination for ions of $\mathrm{Mg}$.

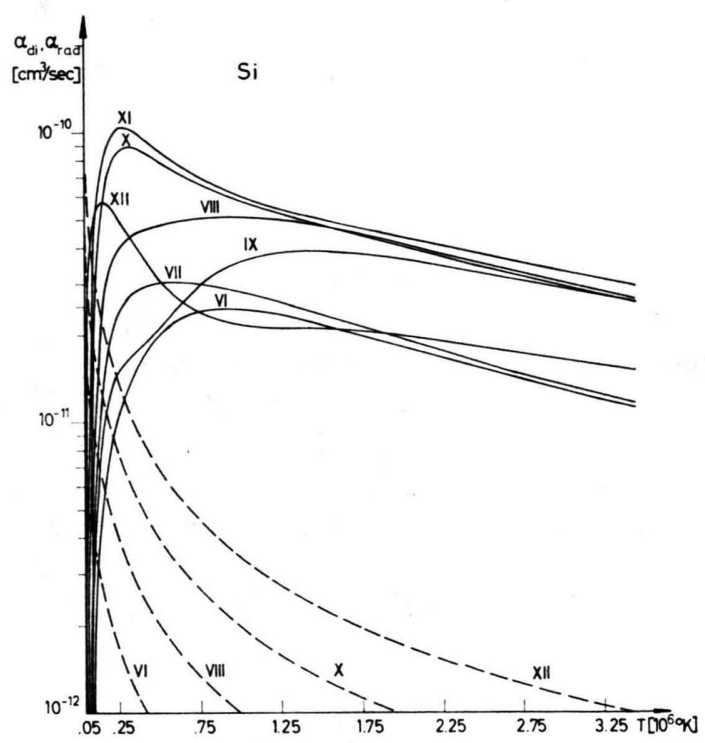

Fig. 9. Coefficients of dielectronic and radiative recombination for ions of Si.

allowed transition of the recombining ion takes place from different atomic shells for two successive stages, the coefficients behave substantially different, for example in case of Ne VIII and Ne IX. Ne VIII contains still one electron in the L-shell, while Ne IX possesses only two K-electrons. From Table 5, one can see that the corresponding wavelengths of the first optically allowed transitions change abruptly. Thus, the maximum of $\alpha_{\mathrm{di}}$ for Ne VIII oc-

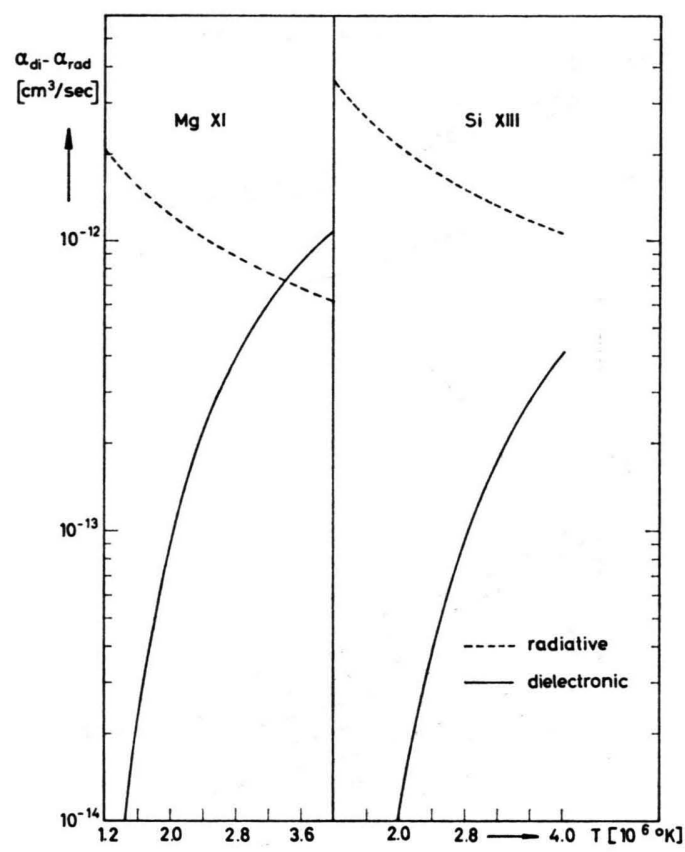

Fig. 10. Coefficients of dielectronic and radiative recombination for high ionization stages of $\mathbf{M g}$ and $\mathrm{Si}$.

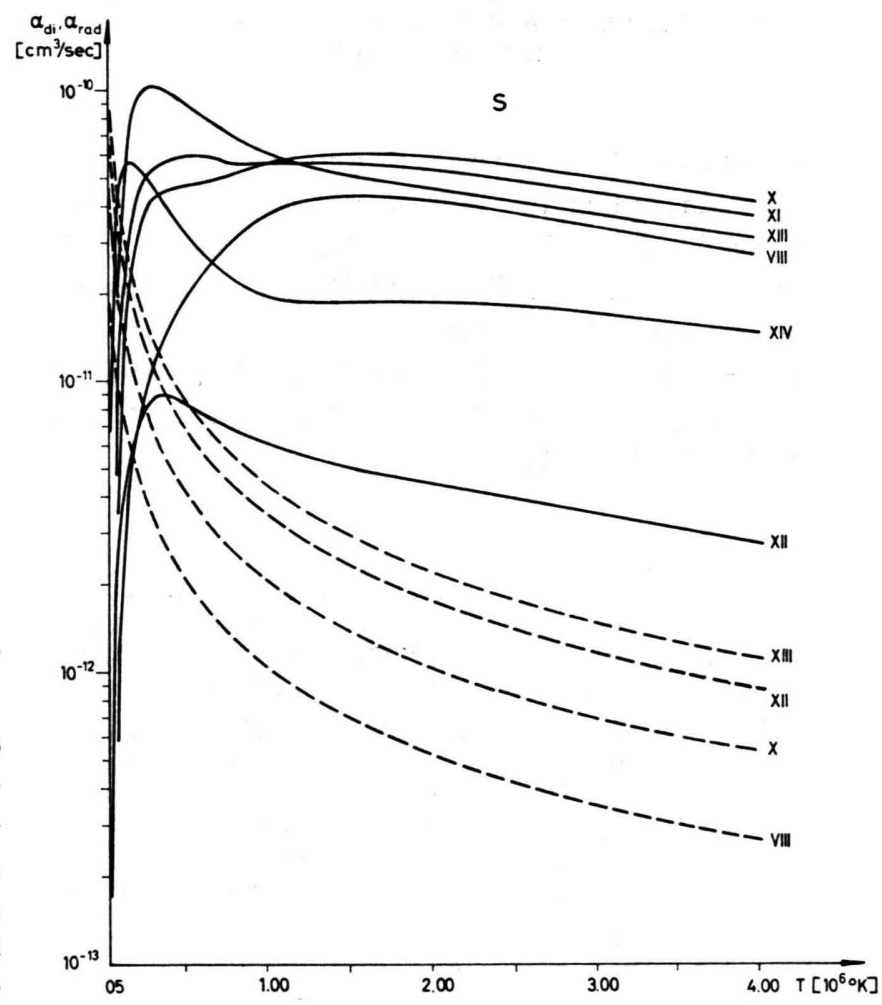

Fig. 11. Coefficients of dielectronic and radiative recombination for ions of $\mathrm{S}$. 


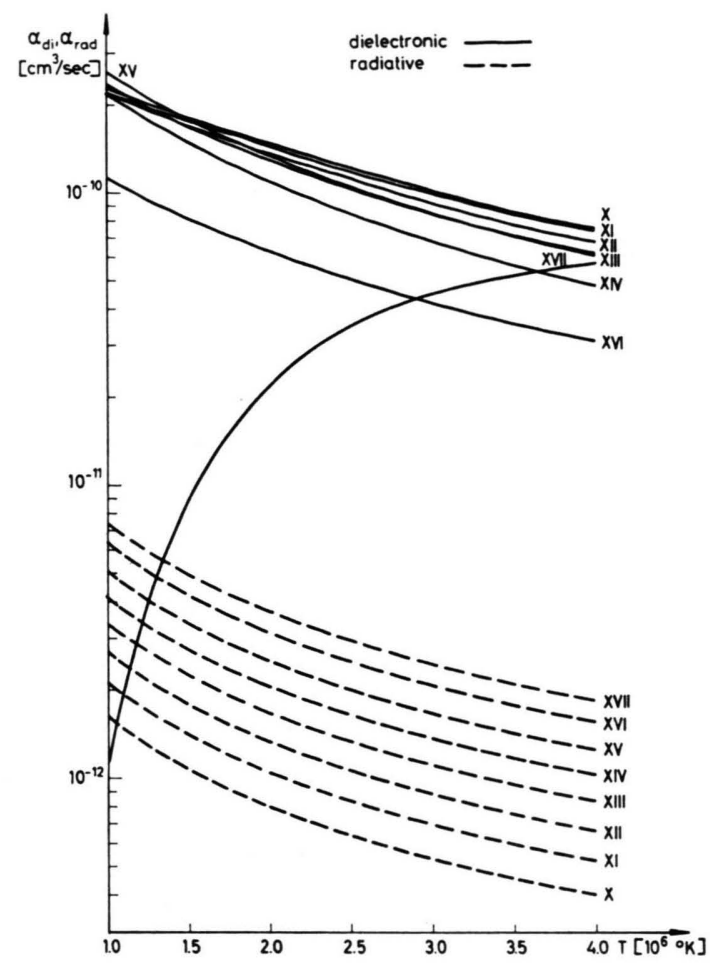

Fig. 12. Coefficients of dielectronic and radiative recombination for ions of $\mathrm{Fe}$.

curs below $10^{6}{ }^{\circ} \mathrm{K}$; the dielectronic recombination is much larger than the radiative recombination. On the other hand for Ne IX (Fig. 7), the maximum in question occurs at $T>4 \cdot 10^{6}{ }^{\circ} \mathrm{K}$, the radiative recombination still predominates up to $2 \cdot 10^{6}{ }^{\circ} \mathrm{K}$. Similar statements can be made for $\mathrm{Mg} \mathrm{X}$ and $\mathrm{Mg} \mathrm{XI}$ (Fig. 8), Si XII and Si XIII (Fig. 9), Fe XVI and Fe XVII (Fig. 12).

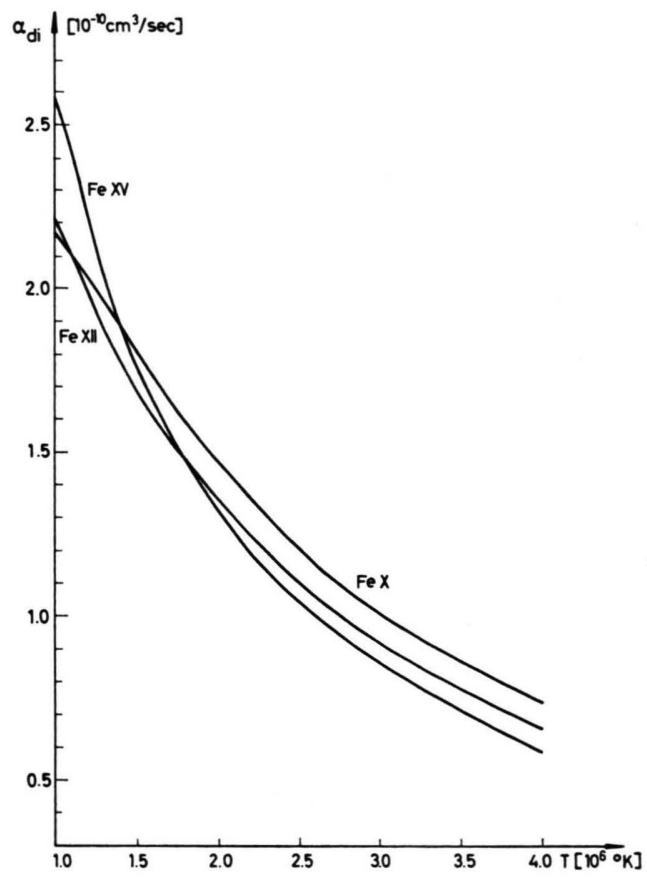

Fig. 13. Coefficients of dielectronic recombination for ions of Fe X, XII, XV (linear scale).

For the ions $\mathrm{Fe} \mathrm{X}$ to $\mathrm{Fe} \mathrm{XIV}$, the wavelength of the first optically allowed transitions lie between about 500 and $200 \mathrm{~A} \mathrm{eV}$; the maxima of $\alpha_{\mathrm{di}}$ occur therefore between 2 and $4 \cdot 10^{5}{ }^{\circ} \mathrm{K}$ (Fig. 12). At temperatures of the order of $10^{6}{ }^{\circ} \mathrm{K}$ and larger, the dielectronic recombination coefficient decreases and is about 50 times larger than the corresponding radiative coefficient. Similar results are obtained for the ions of $\mathrm{Si}$ and $\mathrm{S}$ also, see Figs. 9 and 11.

\begin{tabular}{|c|c|c|c|c|c|c|c|c|c|}
\hline $\begin{array}{l}\text { Ion } \\
\lambda[\AA] \\
t_{\max }\end{array}$ & $\begin{array}{l}\text { C V } \\
40,27 \\
2,23\end{array}$ & $\begin{array}{r}\text { C VI } \\
33,74 \\
2,67\end{array}$ & $\begin{array}{r}\text { N VI } \\
28,79 \\
3,13\end{array}$ & $\begin{array}{r}\text { N VII } \\
24,78 \\
3,63\end{array}$ & $\begin{array}{r}\text { O VII } \\
21,60 \\
4,17\end{array}$ & $\begin{array}{c}\text { O VIII } \\
18,97 \\
4,74\end{array}$ & $\begin{array}{c}\mathrm{Mg} \text { IX } \\
368,1 \\
0,24\end{array}$ & $\begin{array}{r}\operatorname{Mg} \mathrm{X} \\
617,5 \\
0,15\end{array}$ & $\begin{array}{r}\text { Mg XI } \\
9,17 \\
9,81\end{array}$ \\
\hline $\begin{array}{l}\text { Ion } \\
\lambda[\AA] \\
t_{\max }\end{array}$ & $\begin{array}{c}\mathrm{Ne} \text { VII } \\
455,9 \\
0,20\end{array}$ & $\begin{array}{c}\mathrm{Ne} \text { VIII } \\
763,9 \\
0,12\end{array}$ & $\begin{array}{r}\mathrm{Ne} \text { IX } \\
13,38 \\
6,73\end{array}$ & $\begin{array}{r}\text { Si VI } \\
246,0 \\
0,37\end{array}$ & $\begin{array}{l}\mathrm{Si} \text { VII } \\
251,2 \\
0,36\end{array}$ & $\begin{array}{l}\mathrm{Si} \text { VIII } \\
258,4 \\
0,35\end{array}$ & $\begin{array}{l}\text { Si IX } \\
269,0 \\
0,33\end{array}$ & $\begin{array}{l}\text { Si X } \\
281,2 \\
0,32\end{array}$ & $\begin{array}{l}\text { Si XI } \\
303,6 \\
0,30\end{array}$ \\
\hline $\begin{array}{l}\text { Ion } \\
\lambda[\AA] \\
t_{\max }\end{array}$ & $\begin{array}{c}\text { Si XII } \\
510,0 \\
0,18\end{array}$ & $\begin{array}{c}\text { Si XIII } \\
6,65 \\
13.53\end{array}$ & $\begin{array}{l}\text { Fe X } \\
477,4 \\
0,19\end{array}$ & $\begin{array}{c}\mathrm{Fe} \mathrm{XI} \\
458,3 \\
0,20\end{array}$ & $\begin{array}{c}\text { Fe XII } \\
383,1 \\
0,23\end{array}$ & $\begin{array}{c}\text { Fe XIII } \\
312,7 \\
0,29\end{array}$ & $\begin{array}{c}\text { Fe XIV } \\
248,3 \\
0,36\end{array}$ & $\begin{array}{l}\mathrm{Fe} \text { XV } \\
268,7 \\
0,33\end{array}$ & $\begin{array}{c}\text { Fe XVI } \\
348,5 \\
0,26\end{array}$ \\
\hline $\begin{array}{l}\text { Ion } \\
\lambda[\AA] \\
t_{\max }\end{array}$ & $\begin{array}{c}\text { Fe XVII } \\
15,26 \\
5,90\end{array}$ & $\begin{array}{l}- \\
-\end{array}$ & $\begin{array}{l}- \\
-\end{array}$ & $\begin{array}{l}- \\
-\end{array}$ & $\begin{array}{l}- \\
-\end{array}$ & $\begin{array}{l}- \\
-\end{array}$ & $\begin{array}{l}- \\
-\end{array}$ & $\begin{array}{l}- \\
-\end{array}$ & $\begin{array}{l}- \\
-\end{array}$ \\
\hline
\end{tabular}

Table 5. Wavelengths of the first optically allowed transitions. 


\section{Tucker and Gould Formula}

Another formula which is based on a drastic approximation $^{42}$ has been set up by TUCKER and Gould $^{5}$. This very simple formula can be derived directly from the first Burgess formula (4.5). To that end Tucker and Gould

1) employ VAN REgemorTER's ${ }^{44}$ excitation cross section $\sigma_{\mathrm{ex}}$, given by

$$
\sigma_{\mathrm{ex}}=\frac{8 \pi}{\sqrt{3}} \frac{I_{\mathrm{H}^{2}}}{E_{\mathrm{e}}\left(E_{i^{\prime}}-E_{i}\right)} f_{i^{\prime} i} g_{i i^{\prime}} \pi a_{0}^{2},
$$

where $a_{0}=\hbar^{2} / m e^{2}$ is Bohr's hydrogen radius and $g_{i i^{\prime}}$ is an effective Gaunt factor determined from the best observations and calculations. This substitution confines the $l^{\prime \prime}$-dependence only to the statistical weight $\omega\left(n^{\prime} l^{\prime}, n^{\prime \prime} l^{\prime \prime}\right)$ of the doubly excited states.

2) make a very rough approximation in summing over $l^{\prime \prime}$. The statistical weight $\omega$ appears in the second term in the denominator of (4.5). Tucker and Gould substitute this summation by

$\sum_{l^{\prime \prime}} \omega\left(n^{\prime} l^{\prime}, n^{\prime \prime} l^{\prime \prime}\right)=\omega\left(n^{\prime} l^{\prime}\right) \sum_{l^{\prime \prime}}\left(2 l^{\prime \prime}+1\right)=n^{\prime \prime 2} \omega\left(n^{\prime} l^{\prime}\right)$.

3) suppose that the radiation probability

$$
A_{\mathrm{r}}\left(i^{\prime}, i^{\prime \prime} \rightarrow i, i^{\prime \prime}\right) \rightarrow A_{\mathrm{r}}\left(i^{\prime} \rightarrow i\right) \equiv c
$$

i. e. is independent of the quantum numbers of the captured electron. This assumption is also made by BuRgess $^{1}$ and TrefFTz ${ }^{20}$, and was established by SHORE ${ }^{19}$. Deferring the discussion of 2 ) to the end of this section, we sketch first the derivation of the Tucker and Gould formula in the following.

With the assumption 1) the auto-ionization probability $A_{\mathrm{a}}$ can be approximated as

where

$$
\sum_{i^{\prime \prime \prime}} A_{\mathrm{a}}=b n^{\prime \prime-5},
$$

$$
b=1.3 \cdot 10^{18} \frac{z^{2}}{\omega\left(i^{\prime}\right)} \sum_{i^{\prime \prime \prime}} \frac{\omega\left(i^{\prime \prime \prime}\right) f_{i^{\prime \prime \prime} i^{\prime}} g_{i^{\prime \prime \prime} i^{\prime}}}{E_{i^{\prime}}-E_{i^{\prime \prime \prime}}} \sec ^{-1} .
$$

$E_{i}$ and $E_{i^{\prime \prime \prime}}$ are the level energies in $\mathrm{eV}$.

Similarly the numerator of $(4.5)$ can be written as

$$
T^{-3 / 2} a c e^{-E / k T} n^{\prime \prime-3},
$$

with $\quad a=4.942 \cdot 10^{16}\left(\frac{h^{2}}{m k}\right)^{3 / 2} \frac{z^{2} I_{\mathrm{H}}}{E_{i^{\prime}}-E_{i}} f_{i^{\prime} i} g_{i i^{\prime}}$.

42 Compare the remarks of Seaton ${ }^{43}$, TreffTZ ${ }^{28}$ and ShoRE ${ }^{19}$.

43 M. J. Seaton, Atomic Collisions Cross Sections (Section IV: Recombination), Report of Comittee 2b of Commission 14, Transactions of 13th General Asembly of IAU, Prague (D. Reidel, Publ. Co. Holland), esp. p. 242, 1967.
Then one gets finally for the coefficient of dielectronic recombination either the expression (TUCKER and Gould ${ }^{5}$ )

$$
\alpha_{\mathrm{di}}(i, \text { tot })=T^{-3 / 2} \sum_{i^{\prime}} \sum_{n^{\prime \prime}} \frac{a c n^{\prime \prime-3} e^{-E / k T}}{c+b n^{\prime \prime-5}},
$$

or in the form given by $\operatorname{Cox}^{45}$

$=\frac{h^{3}}{(2 \pi m k T)^{3 / 2}} \sum_{i^{\prime}} \frac{\omega\left(i^{\prime}\right) c}{\omega(i)} \exp \left\{-E_{i i^{\prime}} / k T\right\} S\left(i^{\prime}, T\right)$,

where $E_{i i^{\prime}}$ is the excitation energy given by (2.4) and

$$
S\left(i^{\prime}, T\right)=\sum_{n^{\prime \prime}} \frac{n^{\prime \prime 2}}{1+\left(n^{\prime \prime} / n_{\mathrm{c}}\right)^{5}} e^{\left(z^{2} I_{\mathrm{H}} / n^{\prime \prime 2} k T\right)},
$$

$n_{\mathrm{c}}$ being defined by

$$
n_{\mathrm{c}}=26\left(z^{2} g_{i i^{\prime}}\right)^{1 / 5}\left(\frac{I_{\mathrm{H}}}{E_{i i^{\prime}}}\right)^{3 / 5} .
$$

Tucker and Gould then substitute $\sum_{n^{\prime \prime}}$ by an integral, neglecting the exponential factor in (7.8). They obtain the result

where

$$
\alpha_{\mathrm{di}}(\mathrm{TG})=T^{-3 / 2} \sum_{i^{\prime}} C_{i^{\prime}} \exp \left\{-E_{i i^{\prime}} / k T\right\},
$$

$$
C_{t^{\prime}}=\frac{\pi}{5} \operatorname{cosec}\left(\frac{3 \pi}{5}\right) a\left(\frac{c}{b}\right)^{2 / 5} .
$$

For the first excited state the coefficient $C_{i^{\prime}}$ is tabulated in their paper.

According to a comparison of $\alpha_{\mathrm{di}}$ (TG) and the results obtained with Burgess' formula $\alpha_{\mathrm{di}}(\mathrm{B})$ by Cox ${ }^{45}$ for high $z, \alpha_{\mathrm{di}}$ (TG) should be larger than $\alpha_{\mathrm{di}}$ (B) by a constant factor of 3 nearly; this factor rises even higher with the decrease of $z$ up to a value of 8 for $z=1$. Cox expects this rise to be compensated by the $z$-dependence of $g_{i i^{\prime}}$.

In Fig. 14, a plot of $\alpha_{\mathrm{di}}$ (TG) and $\alpha_{\mathrm{di}}(\mathrm{B})$ for Si IX, Si X and in Fig. 15 a plot of $\alpha_{\text {di }}$ for Mg IX and $\mathrm{MgX}$ according to our calculations is given. They show also that for sufficiently low temperatures $\left(t<t_{\mathrm{c}}\right), \alpha_{\mathrm{di}}(\mathrm{TG})>\alpha_{\mathrm{di}}(\mathrm{B})$ which agrees with the estimation of Cox. One notes, however, that at temperatures $t>t_{\mathrm{c}}, \alpha_{\mathrm{di}}(\mathrm{TG})<\alpha_{\mathrm{di}}(\mathrm{B})$, the ratio of the two coefficients decreases with increasing temperature. However, a comparison of the ionization equilibrium for the ions Si IX and Si X, allowing

44 H. van Regemorter, M.N.R.A.S. 121, 213 [1960] ; Astrophys. J. 136, 906 [1962].

45 D. P. Cox, private communication, 1969. 


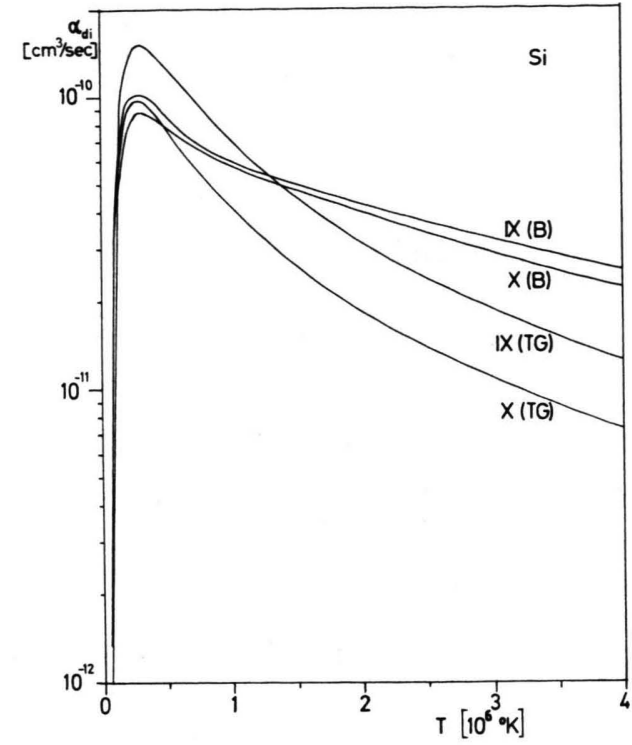

Fig. 14. Comparison of $\alpha_{\mathrm{di}}$ (Burgess) and $\alpha_{\mathrm{di}}$ (Tucker-Gould) for Si IX and Si X.

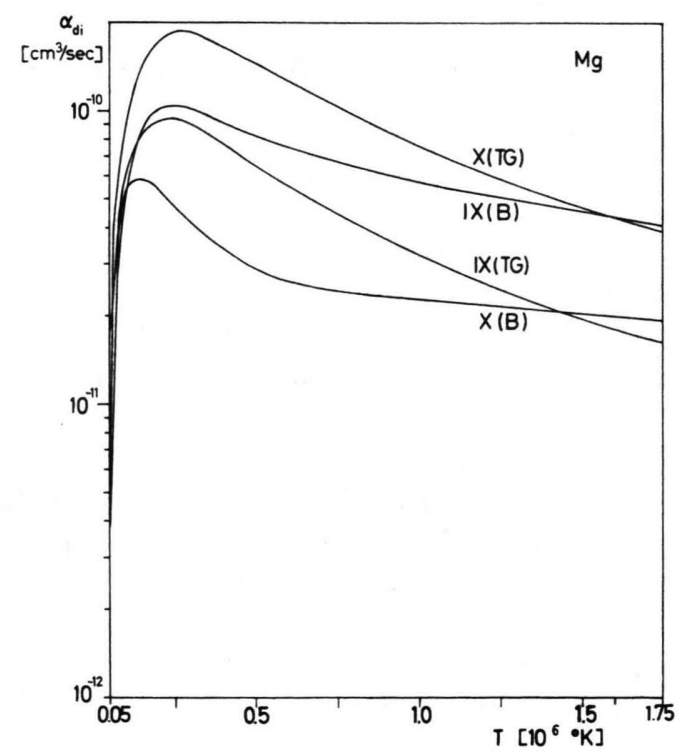

Fig. 15. Comparison of $\alpha_{\mathrm{di}}$ (Burgess) and $\alpha_{\mathrm{di}}$ (Tucker-Gould) for $\mathrm{Mg}$ IX and $\mathrm{Mg} \mathrm{X}$.

for the dielectronic recombination by Tucker and Gould's and by Burgess' formulas (Fig. 21), shows that the populations of the ionization stages as a function of the temperature are not appreciably different from each other.

We may remark that, besides the reasons mentioned before, a further objection to the approxima-

46 G. Elwert, s. Anm. ${ }^{41}$.

47 G. Elwert, Z. Naturforsch. 9 a, 637 [1954].

48 L. BiermanN, Naturwiss. 34, 87 [1947]. tion of Tucker and Gould is the fact that the socalled resonance width

$$
\Gamma_{\mathrm{a}}=\sum_{i} \sum_{l_{\mathrm{e}}} A_{\mathrm{a}}\left(i^{\prime}, i^{\prime \prime} \rightarrow i, E_{\mathrm{e}} l_{\mathrm{e}}\right)
$$

occuring in the denominator of (2.17) turns out to be strongly $l^{\prime \prime}$-dependent when evaluated quantummechanically, especially for sufficiently large $l^{\prime \prime}$; it decreases rapidly with increasing $l^{\prime \prime}$ (SHORE ${ }^{19}$ ). Consequently, the use of $(5.2)$ is very rough. The same conclusion is drawn by Mrs. TrefFTz ${ }^{28}$ also.

\section{Ionization Equilibrium in the Solar Corona}

It can be shown that in the ionization equilibrium of the solar corona the photoionization and the three-body recombination are not predominant (ELWERT ${ }^{46,47}$ ). It was therefore customary to assume that in the solar corona collisional ionization

$$
\mathrm{X}^{+(z-1)}+\mathrm{e} \rightarrow \mathrm{X}^{+z}+\mathrm{e}+\mathrm{e}
$$

on the one hand, and radiative recombination

$$
\mathrm{X}^{+z}(i)+\mathrm{e} \rightarrow \mathrm{X}^{*+(z-1)} \rightarrow \mathrm{X}^{+(z-1)}+h v,
$$

on the other, are the main processes for setting up an equilibrium (BIERMANN ${ }^{48}$, Woolley and ALLeN $^{49}$, Miунамото $\left.^{50}\right)$. Since Burgess' ${ }^{1}$ work we

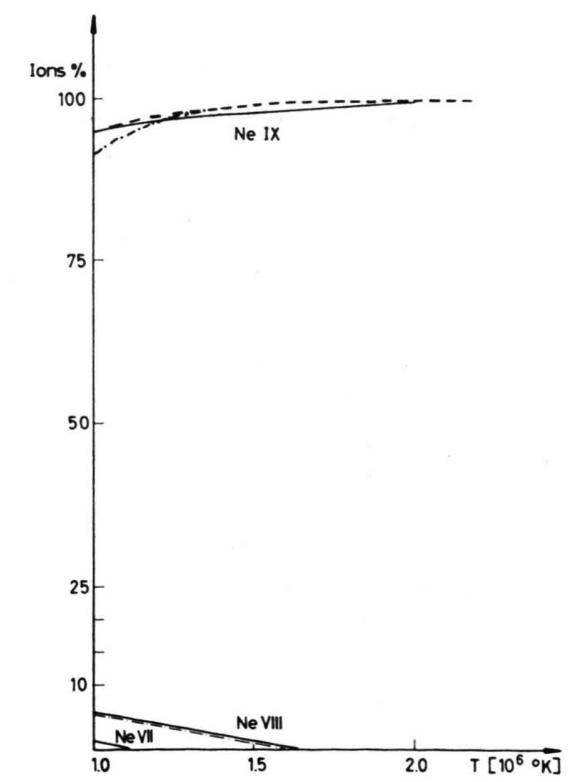

Fig. 19. Ionization equilibrium of Ne-ions; curves as explained in Fig. 16.

49 R. v. D. R. Woolley and C. Allen, M.N.R.A.S. 108, 292 [1948].

50 S. Мгуамото, Publ. Astr. Soc. Pacif. I, 1950. 


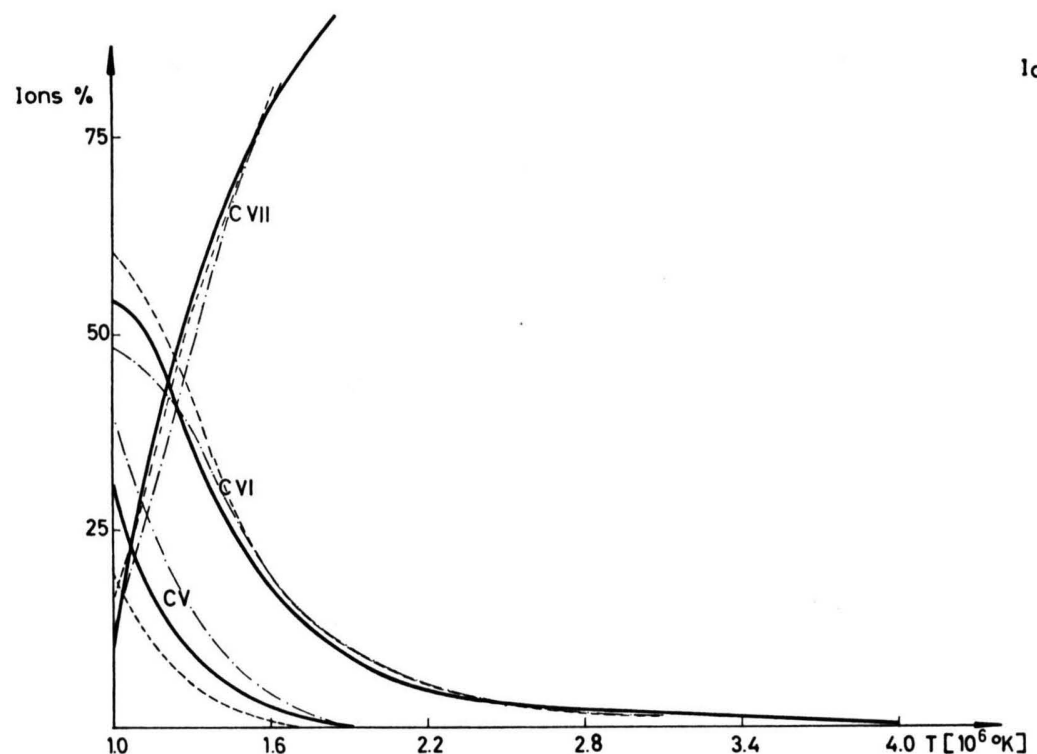

Fig. 16. Ionization equilibrium of C-ions. Present calculation; - - - results of JORDAN ${ }^{22}$ induding density dependent effects; $-.-\cdot-\cdot-$ results of

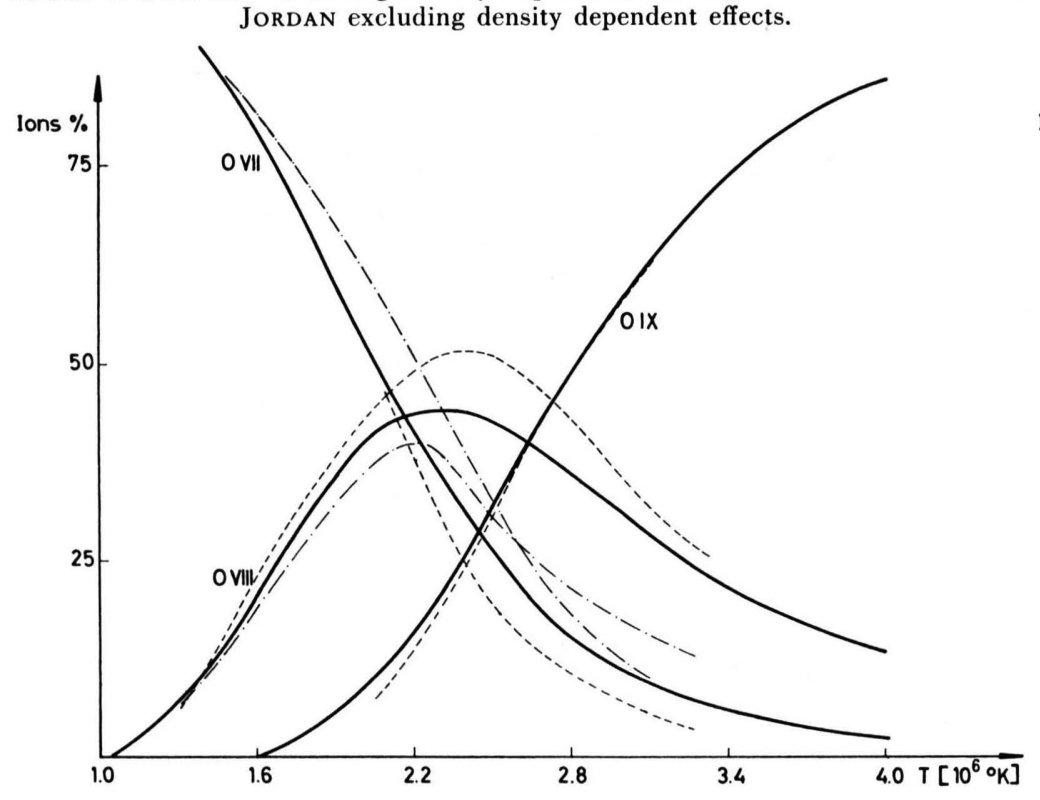

Fig. 18. Ionization equilibrium of O-ions; curves as explained in Fig. 16.

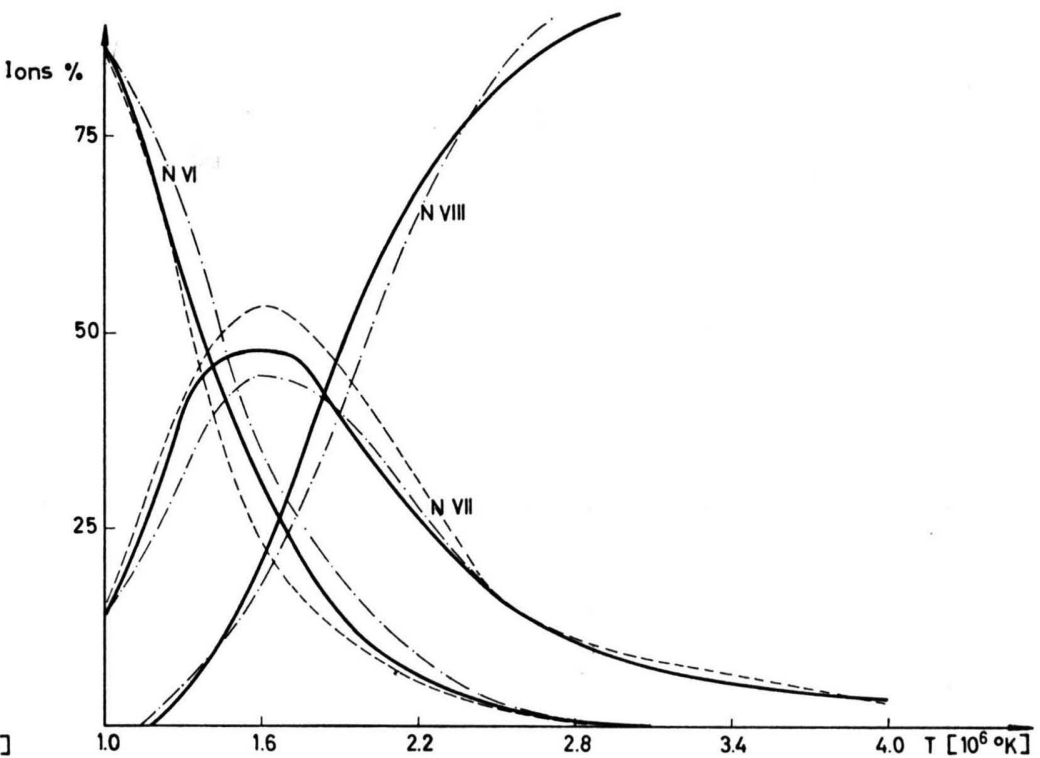

Fig. 17. Ionization equilibrium of $\mathrm{N}$-ions; curves as explained in Fig. 16.

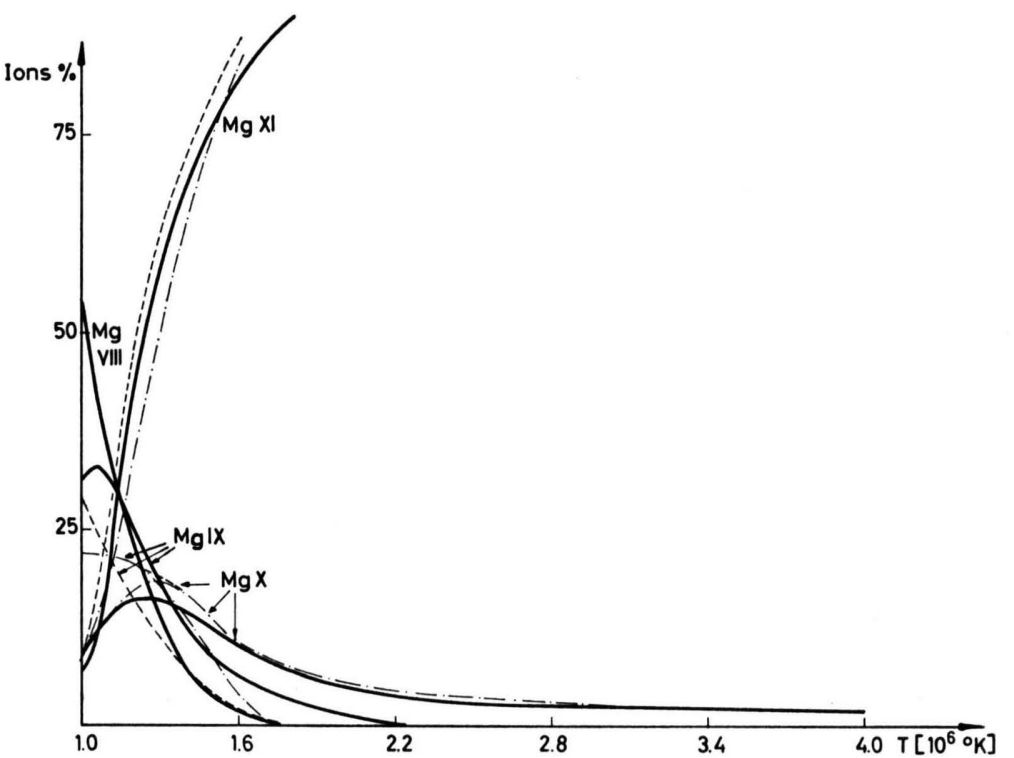

Fig. 20. Ionization equilibrium of Mg-ions; curves as explained in Fig. 16. 


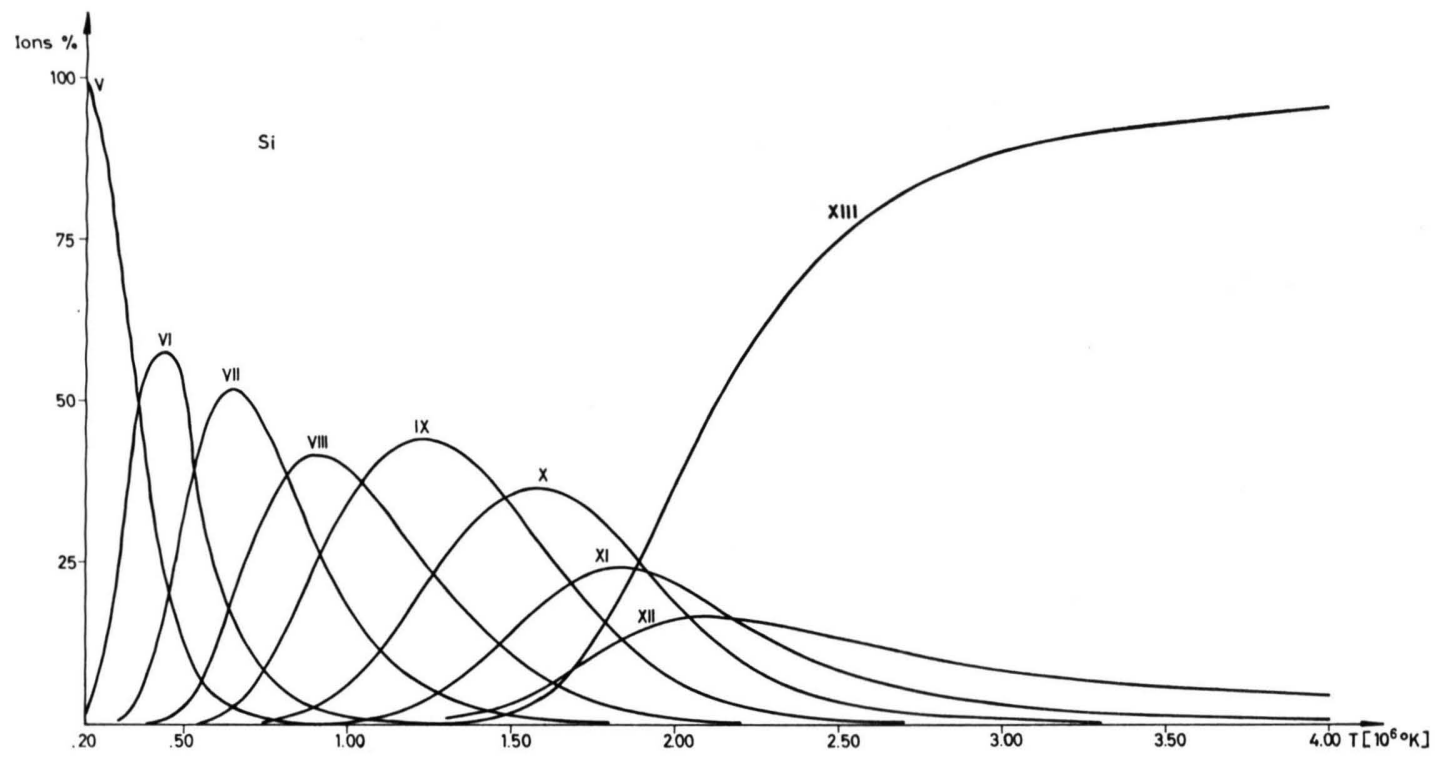

Fig. 21. Ionization equilibrium of Si-ions; present calculation.

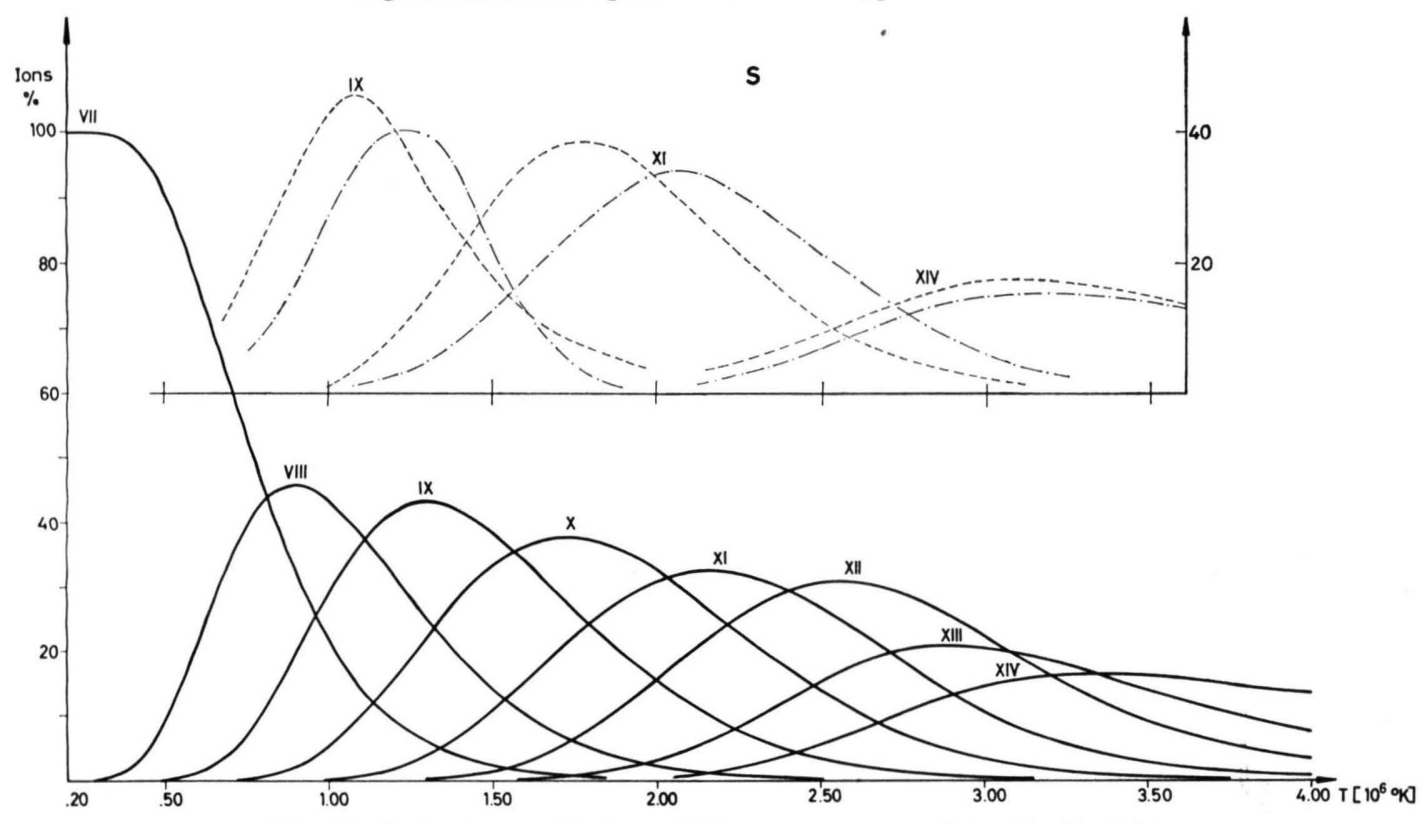

Fig. 23. Ionization equilibrium of S-ions; curves as explained in Fig. 16.

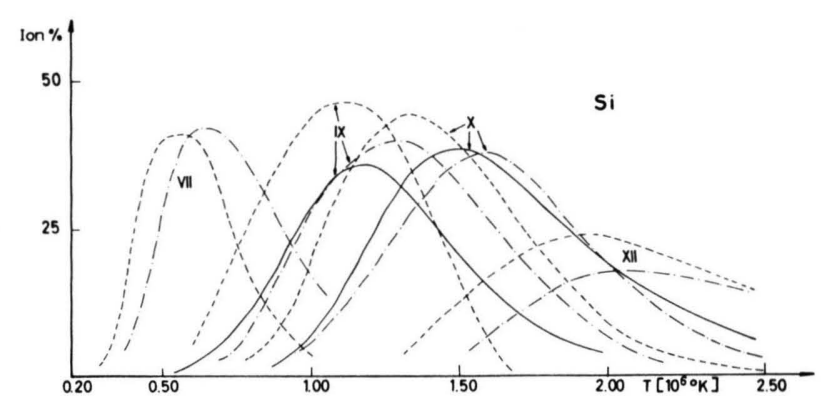

Fig. 22. Ionization equilibrium of Si-ons; curves as explained in Fig. 16 according to JORDAN ${ }^{22}$; full lines according to TUCKER and Gould ${ }^{5}$
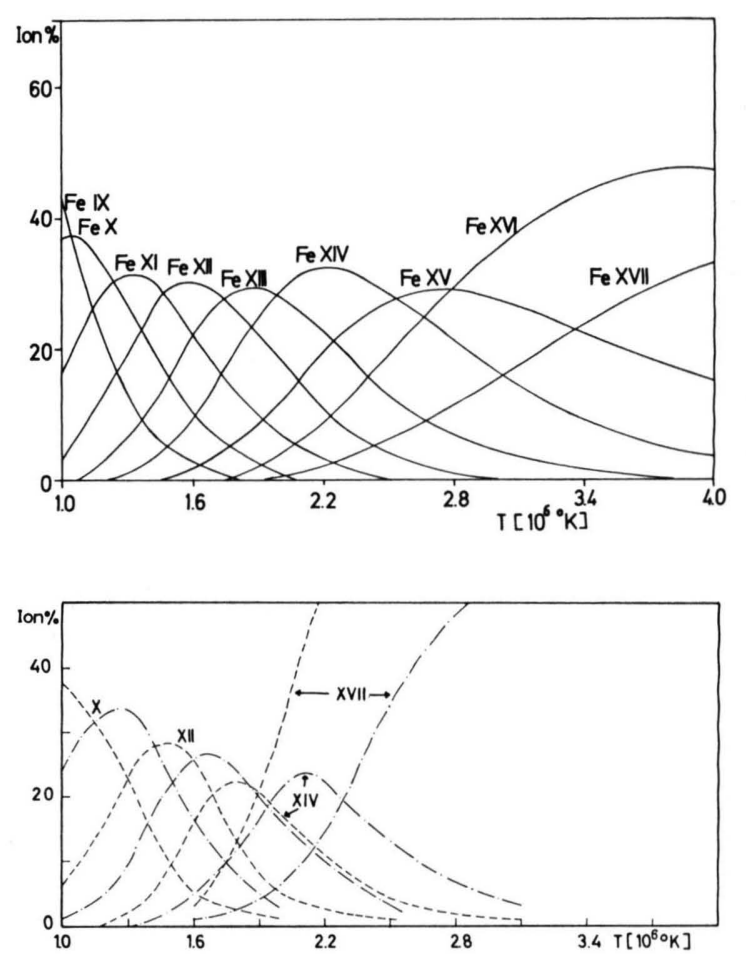

Fig. 24 a and 24 b. Ionization equilibrium of Fe-ions; a) present calculation, b) calculations of JORDAN ${ }^{22}$, curves as explained in Fig. 16. 
know the importance of dielectronic recombination. The total number of collisional ionizations should therefore be equal to the number of dielectronic and radiative recombinations, namely

or

$$
N_{z-1} N_{\mathrm{e}} S=N_{z} N_{\mathrm{e}}\left(\alpha_{\mathrm{rad}}+\alpha_{\mathrm{di}}\right)
$$

$$
N_{z} / N_{\mathrm{z}-1}=S /\left(\alpha_{\mathrm{rad}}+\alpha_{\mathrm{di}}\right),
$$

where $S$ denotes the coefficient of collisional ionization. A sufficiently good expresison for $S$ was first derived by ELWERT ${ }^{46}$, a somewhat simpler formula was then given by SEATON ${ }^{51}$ :

$S\left(\mathrm{X}^{+(z-1)}\right)=2 \cdot 10^{-8} \frac{\zeta_{z-1}}{I^{2} z-1} T^{1 / 2} 10^{-(5040 / T)} I_{z-1} \frac{\mathrm{cm}^{3}}{\mathrm{sec}}$.

$I_{z-1}$ is the ionization potential of $\mathrm{X}^{+(z-1)}$ in $\mathrm{eV}$ and $\zeta_{z-1}$ the number of electrons in the outer shell of $\mathrm{X}^{+(z-1)}$. In Figs. $16-24$ the percentage of specific ion concentration $N\left(\mathrm{X}^{+(z-1)}\right) / N$ is plotted against the temperature, where $N$ is the total number density of all the ionization stages to be considered.

After completing the calculations, some papers were published in which the contribution to the ionization rate by autoionization from collisionally excited levels was considered. Taking into account this density dependent effect, Allen and Dupres ${ }^{52}$ determined the ionization equilibrium. Whereas the concentrations of $\mathrm{O}$ and $\mathrm{Si}$ change little when this autoionization is included, the effect of the increase in the rate of ionization is apparent in the ionization equilibrium of heavy elements (ALLEN and DUPREE ${ }^{52}$ ). It leads to a shift of the maximum of the ions population to lower temperatures, for instance in case of some $\mathrm{Fe}$ ions.

51 M. J. Seaton, Plan. Space Sci. 12, 55 [1964].
Besides the autoionization from the collisionally excited levels, the collisional depopulation of highly excited states should be taken into account (BURGESS and SUMMERS ${ }^{13}$ ), especially in case of high electron densities. A reliable method to include the latter process is in developing stage. Using an estimate of the reduction in dielectronic recombination rate by this density dependent effect, JORDAN ${ }^{22}$ calculated the ionization equilibrium of many ions, using a relation between temperature and density valid under the conditions of the solar atmosphere. For light elements (approximately up to 0 ) the maxima of our ionization curves are close to those of the curves computed by Jordan. Frequently the curves are between those of Jordan calculated with regard to and without regard to density dependent effects, or they even coincide with one of them. For $\mathrm{Si}$ the positions of the maxima differ little as compared to our curves; allowing for density dependent processes, they are shifted a bit towards lower temperatures. This displacement increases for $S$ and is yet more pronounced for $\mathrm{Fe}$.

\section{Acknowledgement}

We would like to express our sincere thanks to Mrs. Dr. E. TreffTZ for a number of useful discussions. Thanks are also due to Drs. W. H. TUCKER and D. P. Cox. - One of us (S. M. R. A.) is indebted to the Bundesministerium für Wissenschaftliche Forschung for financial support. The calculations, reported here, are carried out partly on Siemens 2002 and partly on CDC 3200. We are thankful to the Rechenzentrum der Universität Tübingen for the allotment of the computation time.

52 J. W. Allen and A. K. Dupree, Astrophys. J. 155, 27 [1969]. 